\title{
Neue und bekannte Süd-Asiatische Dipteren
}

\author{
VON \\ D . J. C. H. DE MEIJERE \\ IN HILVERSUM.
}

Hierzu Taf. 8.

\begin{abstract}
Die süd-asiatische Dipterenfauna harrt noch immer der eingehenden Durchforschung. Was uns aus diesem Gebiete erreicht, beschränkt sich einstweilen auf kleine Sendungen oder sogar auf einzelne, zwischen anderen Insekten versteckte Exemplare. Wenig ist jedoch immer besser als nichts, und so habe ich mich entschlossen, auch ein Steinchen zum Gebäude beizutragen und das betreffende, mir zu Gebote stehende Material zu untersuchen. Es besteht dies aus mehreren, in der Sammlung der kgl. zoolog. Gesellschaft Natura Artis Magistra in Amsterdam, in derjenigen des Reichsmuseums zu Leyden ${ }^{1}$ ) und in meiner Privatsammlung befindlichen Stücken. Ein Theil fand sich im Nachlass des Herrn van der Wulp; unter diesen waren besonders auch diejenigen von Bedeutung, welche ihm vor : mehreren Jahren von Herrn R. Oberthür geschenkt wurden ${ }^{2}$ ) und aus Darjeeling im Himalaya-Gebirge (Nord-Bengalen) stammten. Mehrere darunter befindlichen Asiliden wurden derzeit noch von van der Wulp beschrieben ${ }^{3}$ ).

Im Übrigen stammen die meisten unten erwähnten Exemplare aus Niederländisch Ostindien. Namentlich Herrn J. D. Kobus in Pasuruan verdanke ich die Zusendung mehrerer interessanter Formen, unter welchen besonders die von Tosari, einem in 1800 Meter Höhe im Tengger-Gebirge (O. Java) liegenden Orte, von Bedeutung sind. Ich habe die Arten in Uebereinstimmung mit van der Wulp's Catalogue of the described Diptera from South Asia, 1896, angeordnet.

- Im Nachlass von weiland Herrn van der Wulp fand ich überdies noch einige bis jetzt nicht publicirte Beschreibungen ostindischer Dipteren aus der Sammlung des Herrn J. R. H. Neervoort van de Poll zu Rijsenburg. Dieselben wurden von dem leider auch schon verstorbenen Herrn Kannegieter auf Java und Nias erbeutet.

Durch die Freundlichkeit Herrn van de Poll's war ich in der Lage, die Exemplare noch einmal mit den Beschreibungen vergleichen zu können und so die Publication zu ermöglichen. Es freut mich sehr, dazu beitragen zu können, dass die Arbeit der beiden Forscher, welche jeder in seiner Weise sich um die Wissenschaft sehr verdient gemacht haben, nicht vergebens war.

Es sind folgende Arten: Pselliophora nigrithorax und stigmatica, Pachyrrhina fallax, Eriocera nigripennis, Ptecticus tricolor, Rosapha bimaculata. Ich habe sie in der vorliegenden Arbeit an der ihnen systematisch zukommenden Stelle eingefügt, und, behufs der Gleichmässigkeit, die Beschreibungen van der Wulp's in's Deutsche übersetzt.
\end{abstract}

1) Zwei neue Arten aus diesem Museum beschrieb ich vor Kurzem gesondert in: Notes from the Leyden Museum XXIV. 1903. p. 177-178, Note V. Es sind Helophilus caudatus nnd Pseudoformosia pauper.

2) Man vergl. Tijdschr. v. Entomol. XXXIX. 1896. p. 95.

3) Van der Wulp. Aanteekeningen betreffende Oost-Indische Diptera. VII. Asilidae. Tijdschrift v. Entomol. XLI, p. 115. 


\section{BIBIONIDAE}

Bibio obscuripennis n. sp. $2 \sigma^{\pi} \sigma^{\pi}, 1$ 옹 Darjeeling (Bengalen), A. Desgodins leg.

б Ganz schwarz, fast matt; auch die Behaarung von dieser Farbe. An der Unterseite des Abdomens ist dieselbe ziemlich lang. Auch die Fühler, der Rüssel, die Taster, die Schwinger und die Beine sind schwarz. Fühler kurz, bedeutend kürzer als die Taster.

Von den Dornen am Ende der Vorderschienen ist der äussere doppelt so lang wie der innere; auch ersterer ist jedoch relativ kurz, nur etwa $1 / 5$ des Metatarsus lang; die Farbe ist schwarz oder rothbraun, das Ende ist stumpf.

Hinterschenkel in der Endhälfte verdickt, am Ende jedoch wieder verjüngt; die Hinterschienen erweitern sich allmählich. Flügel einfarbig graubraun; die vorderen Adern dunkelbraun, die hinteren blass, das Stigma lang und schmal, schwarzbraun. Das Wurzelstück der Cubitalader doppelt so lang wie die kleine Querader. Das Geäder ähnelt sehr dem von B. Marci, aber letztere Querader ist kürzer und weniger schief gestellt.

Körperlänge $13 \mathrm{~mm}$. , Flügellänge $11 \mathrm{~mm}$.

. Beim $q$ ist der Thorax noch kürzer behaart als beim $\sigma^{\top}$, die Vorderschienen sind kürzer und dicker, die Dorne relativ grösser, etwa halb so lang wie der Metatarsus. Flügellänge $12 \mathrm{~mm}$.

Die Art ähnelt dem B. Marci, das $\sigma^{\top}$ unterscheidet sich aber sofort durch die ganz braunen Flügel, das $\$$ durch die viel kürzere Behaarung.

Plecia fulvicollis F. 1 ㅇ Tosari (Java), Kobus leg.

P lecia lugubris n. sp. Tàtsiénloû (Thibet), $4 \sigma^{7} \sigma^{7}$, Mgr. F. Biet leg.

Ganz mattschwarz; Thorax mit zerstreuter, sehr kurzer, gelblicher Behaarung; der Hinterleib etwas länger, aber ebenfalls hell behaart. Zangenglieder gleichmässig gekrümmt, am Ende spitz. Beine glänzend schwarz, wenig behaart, mit 3 Haftläppchen, Hinterschenkel vor dem Ende etwas verdickt, Hinterschienen nach der Spitze hin allmählich etwas erweitert. Metatarsen fast halb so lang wie die Schienen und von der Dicke des Schienenendes, etwas dicker als die folgenden Tarsenglieder. Flügel gleichmässig dunkel, die Gabelzelle in der Mitte der Flügel gestielt (wie es bei den meisten Plecia-Arten der Fall ist, jedoch nicht bei Pl. melanaspis), der Stiel halb so lang wie die Arme. Oberer. Zweig der 3 ten Längsader ziemlich lang und dem unteren fast parallel verlaufend, (wie bei P1. melanaspis, nur ist hier der obere Zweig noch länger).

Durch die Abwesenheit rothgefärbter Partien stimmt die Art mit Pl. tristis v. d. Wulp überein; letztere hat aber den Hinterleib und 3 Längsstriemen auf dem Thorax von glänzend schwarzer Farbe.

Bei den amerikanischen, ganz schwarzen Arten Pl. funebris F. und Pl. plagiata Wied. zeigen die Flügel nur um die Adern dunkle Säume.

Körperlänge $7 \mathrm{~mm}$; Flügellänge $6 \mathrm{~mm}$.

Plecia melanaspis Wied. 'Tosari (Java), zahlreiche Exemplare (Kobus leg.); Darjeeling (Ben. galen) $1 \sigma^{\top}$ und 3 우 (A. Desgodins leg.).

Bei den Exemplaren von Darjeeling wird die Grenze zwischen der vorderen, schwarzen, und der hinteren, rothen Thoraxhälfte meistens durch eine fast gerade Linie gebildet; bei den javanischen setzt sich das Schwarz in der Mitte etwas weiter fort als an den Seiten, sodass daselbst die Ahgrenzung wenig scharf ist. 
Von Thibet (Tàtsiénloû) liegt mir ein von Mgr. F. Biet gesammeltes Fragment einer mit Pl. melanaspis sehr übereinstimmenden Plecia vor, wahrscheinlich ein $\sigma^{7}$. Die Flügel sind aber dunkler, einfarbig rauchbraun. Dazu ist die Gabel in der Flügelmitte sehr kurz gestielt, während sich bei PI. melanaspis die Querader höchstens an der Wurzel der Gabel, meistens aber etwas vor derselben befindet. In dieser Hinsicht verhalten sich jedoch auch die BibioArten nicht immer constant.

Wiedemann's Beschreibung von Pl. japonica ist viel zu kurz um zu entscheiden, ob hier vielleicht diese Art vorliegt oder ob sie von Pl. melanaspis verschieden ist.

\section{TIPULIDAE.}

Ps elliophora insignis n. sp. Tosari (Java), $1 \sigma^{7}$, Kobus leg.

Matt sammetschwarz, der $1^{\text {te }}$ Hinterleibsring ausser der Wurzel und einem dreieckigen Fleckchen an der Rückenseite nahe dem Hinterrande gelb; der 2te Ring jederseits mit einem dreieckigen gelben Flecken und auch die Unterseite grösstentheils gelb.

Fühler schwarz, zu beiden Seiten lang gekämmt, die Anhänge ca $21 / 2$ mal so lang als das Glied. Das 2te Fühlerglied sehr kurz, das dritte unten am Ende mit grossem Zahn.

Beine schwarz, die Vorderschenkel an der Wurzelhälfte, die Mittelschenkel bis auf das Enddrittel, die Hinterschenkel bis auf die Spitze gelb.

Flügel dunkelbraun mit gelber Wurzel und hellerem, nicht sehr scharf begrenztem Flecken vor dem wenig verdunkelten Stigma. Die Gabel an der Flügelspitze kurzgestielt.

Hypopyg mattschwarz, obere Lamellen desselben eirund.

Körperlänge $17 \mathrm{~mm}$; Flügellänge $18 \mathrm{~mm}$.

Sehr nahe verwandt erscheint die nur im weiblichen Geschlechte bekannte P. annulosa v. d. Wulp. Diese hat jedoch ganz schwarze Schenkel, die Hinterleibsbasis ist orangefarbig und die Flügel zeigen keine gelbe Wurzel. Durch die ganz schwarzen Schienen stimmt sie mit der vorliegenden Art überein.

Es möge hier gleichzeitig bemerkt werden, dass die in der Beschreibung dieser Art angegebenen helleren Stellen in der verdunkelten Flügelfläche kein specifisches Merkmal bilden, denn nach einer handschriftlichen Mittheilung im Nachlass van der Wulp's sah dieser Autor ein von Fruhstorfer gesammeltes $ᄋ$ von Pengnengan (Java), in der Sammlung van de Poll, welches sich durch die überall sehr dunklen Flügel von der Type unterschied; nur ein Wisch vor dem schwarzen Randflecken war etwas heller.

Pselliophora nigrithorax n. sp. (van der Wulp descr.). Tjibodas, Gede (Java), 1 ơ', Kannegieter leg.; Sukabumi (Java), 1 q, Fruhstorfer leg.

Der Ps. ardens Wied., compedita Wied. und fuscipennis Macq. (= javanica Dol.) ähnlich, aber durch den ganz schwarzen Thorax verschieden, von den beiden letzteren auch dadurch, dass den Vorder- und Mittelschienen ein weisser Ring abgeht.

Kopf orangegelb, ein Flecken an Hinterkopfe und die dem hinteren Augenrande benachbarte Partie schwarz. Fühler des $\sigma^{\top}$ schwärzlich, länger als Kopf und Thorax, das 3te Glied unten mit

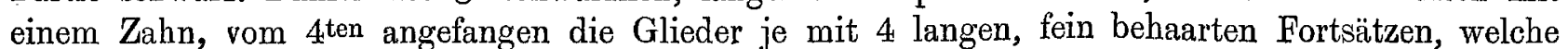
fast $3 \mathrm{mal}$ so lang wie das betreffende Glied sind; beim $\&$ sind die Fühler kaum so lang wie der Kopf, rothbraun, an der Spitze schwarz, das 1 te Glied verdickt, das 2 te sehr kurz, becherförmig, das 3 te fast solang wie das 1 te; die folgenden kurz und breit.

Taster gelblich, beim or verdunkelt.

Thorax und Schildchen einfarbig schwarz, oben am Prothorax des $\sigma^{7}$ ein gelbes Fleckchen, welches jedoch dem $Q$ fehlt; vom Hinterleib sind in beiden Geschlechtern die 3 ersten Ringe gelb 
oder röthlichgelb, der übrige Theil schwarz; das Hinterleibsende des $\sigma^{\pi}$ kolbenartig, wie abgestutzt, die Legescheide des O klein, dornartig.

Beine schwarz, die Wurzelhälfte der Vorder- und Mittelschenkel rothgelb, die Hinterschienen nahe der Wurzel mit einem weissen Ringe.

Flügel an der Wurzel orangegelb, welche Färbung beim $\sigma^{\top}$ noch nicht $1 / 3$ der Flūgellänge einnimmt, weiter schwarzbraun mit einem gelben Flecken gegen den Vorderrand und lichteren Flecken in mehreren Zellen. Beim $q$ hat sich die gelbe Farbe mehr ausgedehnt und beträgt fast $2 / 3$ der Flügellänge; die braune Farbe der Spitze überschreitet noch eben die obere Grenze der Discoidalzelle und an der vorletzten Längsader findet sich ein dunklerer Schatten, welcher sich gegen den Hinterrand erweitert. Die Gabelzelle an der Flügelspitze ist beim $\sigma^{\top}$ fast ungestielt, beim $q$ sind die beiden Aeste ganz gesondert; die 5te Hinterrandzelle an ihrer Ausmündung merkbar verschmälert.

Schwinger gelb.

Körperlänge $\sigma^{7} .17 \mathrm{~mm}$; Flügellänge $18 \mathrm{~mm}$.

» $14 \mathrm{~mm}$; $\quad 14 \mathrm{~mm}$.

Diese Art gleicht sehr der vorigen, ist aber durch folgende Merkmale verschieden: die Fühler sind an der Wurzel gelb, der Kopf ist grösstentheils und auch die Taster sind gelb, der Prothorax zum Theil, der Hinterleib an der äussersten Wurzel von dieser Farbe; die Hinterschenkel sind durchaus schwarz, die Hinterschienen zeigen einen weissen Ring. Bei Ps. insignis dagegen ist der Kopf mit seinen Anhängen und der Prothorax ganz schwarz, ebenso die Wurzel des Hinterleibes; die Hinterschenkel sind bis auf die Mitte gelb und an den Schienen fehlt der weisse Ring.

Auch im Hypopyg finden sich Unterschiede. Die oberen Anhänge sind bei Ps. nigrithorax spitzer, die Hinterseite des Hypopygs ist bei dieser Art glänzender, mit weisslichem Schimmer und zeigt in der Mitte 2 röthlich-gelbe Stellen; bei Ps. insignis ist dasselbe matt, dichter behaart, ganz schwarz, der demselben vorangehende Ring zeigt unten in der Mitte des Hinterrandes braunröthliche Behaarung.

Pselliophora stigmatica n. sp. (van der Wulp descr). Lahago, Central-Nias, 1 \&, Kannegieter leg. - Fig. 1.

Gelb, mit schwarzer Zeichnung, Fühler und Beine schwarz; Hüften und Schenkel, letztere mit Ausnahme der Spitze, gelb; Schienen mit weissem Ringe nahe der Wurzel. Flügel mit braunem Randflecken.

Kopf gelb; rings um den Hinterkopf ein breites, ringförmiges, schwarzes Band. Fühler etwas länger als der Kopf, schwarz; das 1te Glied cylindrisch, das 2te kurz, becherförmig; das 3te und die folgenden Glieder unten etwas sägeartig erweitert; Taster schwarzbraun. Thorax gelb, auf dem Rücken vor der Quernaht mit 3 schwarzen Längsstriemen, von welchen die mittlere sich vorn erweitert, den Prothorax jedoch freilässt; hinter der Quernaht findet sich noch ein $V$-förmiger schwarzer Flecken; ferner tragen die Brustseiten in der Mitte noch je ein schwarzes Fleckchen. Schildchen schwärzlich; Hinterrücken gelb, hinten mit grossem, rundem, schwarzem Flecken.

Hinterleib gelb, am 2ten und den folgenden Ringen mit schwarzer Querbinde; die des 2 ten Ringes bildet ein Dreieck mit nach vorn gerichteter Spitze; die der folgenden Ringe sind hinten in der Mitte etwas ausgebuchtet; die letzten Ringe und die gerade, hinten zugespitzte Legescheide sind glänzend schwarz, letztere am äussersten Ende rothgelb. Bauch fast bis zum Ende gelb, mit schmalen, schwarzen Vorderrändern.

Beine mässig lang, nicht besonders stark; Hüften und Schenkel gelb, bei letzteren das Enddrittel schwarz; Schienen und Tarsen schwarz; erstere nahe der Wurzel mit einem weissen Ringe.

Flügel glasartig, mit blassgelbem Anflug, welcher längs dem Vorderrande sich etwas mehr hervorhebt; die Spitze etwas verdunkelt, das Stigma braun; die Gabelzelle aus der Discoidalader kurz gestielt.

Schwinger gelb, mit dunklerem Stiel.

Körperlänge $15 \mathrm{~mm}$., Flügelläng'e $14 \mathrm{~mm}$. 
Nach den weiss-geringten Schienen zu urtheilen d̈̈rfte die Art wohl zur Gattung Ps ellioph ora gehören, auf welches Merkmal gerade dieser Name hindeutet. Auch die Form des $3^{\text {ten }}$ Fühlergliedes, welches sich von unten nach oben allmählich etwas erweitert und diejenige der Fühler überhaupt sprechen dafür. Doch liesse das in der Mitte verbreiterte Abdomen und die nicht verdunkelten oder buntgefärbten Flügel vermuthen, dass wir es hier mit einer Ctenophora zu thun hätten. Nur die Untersuchung des Männchens könnte hier die Entscheidung bringen, weil die Unterschiede zwischen den Weibchen beider Gattungen einstweilen ziemlich geringfügig sind.

Tipula monochroa Wied. Tosari (Java), 1 Ex., Kobus leg.

Ausser den 3 Längsstriemen finden sich hinter der Quernaht des Thorax noch jederseits 2 dunklere Flecken.

Tipula praepotens Wied. Tosari (Java), $1 \sigma^{\prime}$, Kobus leg.

Tipula thibetana n. sp. Tàtsiénloû (Thibet) $3 \sigma^{7} \sigma^{7}$, Mgr. F. Biet leg. - Fig. 2, 3.

Eine sich sofort durch seine Grösse auszeichnende Art mit marmorirten Flügeln.

Kopf grau, auf der Stirne eine schwarze Längsstrieme, Stirne oberhalb der Fühler vorgewölbt. Die Schnauze relativ lang.

Fühler grau; das 1 te Glied lang, das 2 te sehr kurz, gelb, die folgenden verjüngen sich allmählich, sie sind grau, nur ganz am Ende gelblich.

Prothorax grau, in der Mitte schwarzbraun; Mesothorax vor der Quernaht braungelb mit 3 sehr breiten, dunkelbraunen Längsstriemen, sodass von der Grundfarbe nur 2 schmale, vorn etwas erweiterte Streifen übrig bleiben, welche den Prothorax nicht erreichen, indem die 3 Striemen vorn zusammenhängen. Auch vor der Quernaht ein schmaler, braungelber Saum. Die 3 Striemen sind an den Rändern kastanienbraun eingefasst, über die mittlere verläuft eine Medianlinie von derselben Farbe. Die Seitenstriemen sind im vordern Theil fast ganz kaffeebraun.

Hinterer Theil des Thorax aschgrau, am Hinterrücken an den Seiten breit dunkelbraun schimmernd, sodass bei bestimmter Ansicht nur ein dreieckiger mittlerer Theil, dessen Spitze nach vorn gerichtet ist, aschgrau erscheint. Vor dem Schildchen eine lichtgraue, beiderseits schwarzbraun begrenzte Längsstrieme, Brustseiten aschgrau, ebenfalls hin und wieder mit dunkelbraunem Schimmer.

Hinterleib braun, der le Ring aschgrau, die Vordersäume der Ringe bisweilen heller; am Seitenrande je eine wenig auffällige dunklere Strieme.

Hypopyg (Fig. 3) ziemlich gross, namentlich hoch, von mattbrauner Farbe, mit gelblicher Behaarung. Von den Anhängen sind 2 obere, einander kreuzende am meisten auffällig; dieselben enden spitz und zeigen an der lnnenseite einen stumpfen Zahn; unter denselben liegen 2 gelbbehaarte, gerundete Läppchen, noch weiter nach.. unten 2 langbehaarte, stiftförmige Vorsprünge.

Beine braun, Tarsen dunkler.

Flügel marmorirt, grösstentheils graulich, am Vorderrande schmal gelbbraun; Stigma gross, dunkelbraun, ein ebensolches Fleckchen an der Wurzel der Radialader; weiter mit mehreren relativ kleinen, weisslichen Flecken und Streifen, u. A. einem in und mehreren rings um die Discoidalzelle, einem V-förmigen, bisweilen in mehrere Theile aufgelösten an der Flügelspitze, und 2 mehr oder weniger getheilten, im Zickzack verlaufenden Streifen auf der Flügelmitte. Adern gelblich.

Schwinger braungelb mit dunkelbraunem Knopfe.

Körperlänge $26 \mathrm{~mm}$.; Flügellänge $29 \mathrm{~mm}$..

Pachyrrhina dorsalis n. sp. Tosari (Java), 1 \&, Kobus leg.

Kopf braungelb; Taster schwarzbraun; auf der Mitte des Rüssels ein schwarzes Strichelchen. Fühler schwarzbraun, die beiden Wurzelglieder hellgelb. Am Hinterkopf findet sich ein schwarzbrauner, weisslich schimmernder Flecken, welcher die Stirne bei weitem nicht erreicht und vorn stumpf endet. 
Stirne gelb, nur hinten am Augenrand etwas verdunkelt. Thorax braungelb, die Oberseite mit 3 sehr breiten, glänzend schwarzen, weisslich schimmernden, fast verschmolzenen Längsstriemen, von welchen die mittlere sich bis zum Prothorax erstreckt; das Schildchen und ein davor befindlicher Längsstreifen heller, braungelb, auch der Hinterrücken nur am Ende schwarzbraun. Thoraxseiten bräunlich-gelb, der Bauch etwas verdunkelt.

Hinterleib bräunlich-gelb, mit einer am 2en Ringe anfangenden, breiten, schwarzen Längsstrieme und schmäleren, schwarzen Seitenstriemen; die beiden letzten Ringe durch Zussammenfliessen derselben ganz schwarz.

Legeröhre mässig verlängert, bräunlich-gelb, gerade.

Vorderbeine bräunlich-gelb, die Tarsen gegen das Ende zu allmählich dunkler.

Flügel mit schwarzbraunem Stigma; die Discoidalzelle klein, noch nicht halb so lang wie die obere, aus derselben entspringende Ader. Körper- und Flügellänge $14 \mathrm{~mm}$.

Pachyrrhina fallax n. sp. (van der Wulp descr.). Buitenzorg und G. Tji Salimar in WestPreanger (Java), $1 \sigma^{\top}$ und 2 \&Q, Kannegieter leg.

Kopf orangegelb, ohne Flecken, nur mit einer bräunlich schwarzen Längsstrieme auf dem Untergesicht. Fühler des $\sigma^{7}$ etwas länger als Kopf und Thorax; die beiden Wurzelglieder gelb, der übrige Theil schwarz; das 3te Glied ist cylindrisch und überhaupt das längste; die 4 oder 5 folgenden Glieder sind unten tief eingebuchtet, die letzten cylindrisch und allmählich verdünnt. Beim $Q$ sind die Fühler kürzer und einfach. Taster gelblich.

Thorax hell schwefelgelb, am Rücken vor der Quernaht mit 3 glänzend schwarzen Striemen, die mittlere vorn erweitert und bis zum Prothorax fortgesetzt; die beiden Seitenstriemen sind vorn abgekürzt und daselbst nicht nach unten gebogen; hinter der Quernaht noch ein Paar breite n-förmige, schwarze Striemen. Prothorax an den Seiten schwarz; Brustseiten mit einem dreieckigen, schwarzen Flecken oberhalb der Mittelhüften; Schildchen glänzend schwarz, beim $q$ bräunlich; Hinterrücken zu beiden Seiten von einem schwarzen Streifen eingefasst, gelb, mit einem grossen, schwarzen Flecken am Hinterrand.

Hinterleib rothgelb, die Hinterränder der 4 ersten Ringe, sowie der ganze $5^{\text {te }}$ und 6 te Ring, schwarz; der 7te Ring und die äusseren Genitalorgane rothgelb. Legescheide gerade.

Hüften und Schenkel rothgelb, letztere an der Spitze, sowie die Schienen und Tarsen schwarz oder schwarzbraun.

Flügel hyalin, mit mässig dunklem, braunem Stigma.

Schwinger gelb, mit dunklerem Stiel.

Körperlänge $11 \mathrm{~mm}$. , Flügellänge $10 \mathrm{~mm}$.

Pachyrrhina dimidiata n. sp. Neu-Guinea, 1 \&, R. Semon leg. - Fig. 4.

Das Exemplar sieht P. melanura Ost. Sack. ${ }^{\text {) }}$ ) ähnlich, der Thorax zeigt aber eine andere Zeichnung. Der Prothorax ist an den Seiten des Hinterrandes schwarz. Hinter demselben finden sich 3 zusammengeflossene, mattschwarze Längsstriemen, von. welchen die mittlere sich bis zum Prothorax erstreckt, hinten aber die Quernaht nicht überschreitet; die seitlichen sind vorn verkürzt und erstrecken sich hinten bis zum Schildchen. Die mittlere Strieme zeigt noch einen glänzend schwarzen, dreieckigen Flecken; derselbe nimmt hinten fast die ganze Breite der Strieme ein, verjüngt sich nach vorn allmählich und endet spitz in kurzer Entfernung vom Prothorax.

Das Schildchen ist schwarz, der Hinterrücken nur am Ende von dieser Farbe.

Die schwarze Endhälfte des Abdomens ist überall glänzend, also ohne sammtartige Binden wie bei P. melanura.

Die Beine sind heller, als bei letzterer. Schenkel und Schienen bräunlichgelb, am Ende verdunkelt.

Pach yrrhina scurroides n. sp. Tosari (Java), $3 \%$, Kobus leg.

Die Art sieht der P. scurra ähnlich, das Flügelstigma ist wie bei dieser blassbraun, der

1) Osten Sacken. Ann. Mus, Genova XVI. p. 401. 
Scheitel aber ganz matt, der Hinterleib weniger verlängert und die Seitenstriemen des Thorax setzen sich weniger weit über die Quernaht fort.

Fühler schwarz, die 2 Wurzelglieder gelb; Taster braun, an der Wurzel dunkler. Stirn und Scheitel mattgelb, letzterer vorn etwas gebräunt; vom Halse angefangen verläuft nach vorn hin ein schmaler, matter, dunkelbrauner Streifen, welcher an der Stirne spitz endet. Rüssel vorn etwas verdunkelt.

Prothorax gelb; Thorax mit 3 glänzendschwarzen Längsstriemen, die mittlere ist vorn etwas erweitert, verläuft vom Prothorax bis etwas über die Quernaht und endet spitz. Die Seitenstriemen sind nicht nach vorn umgebogen, dicht hinter der Quernaht sind sie an der Medianseite plötzlich erweitert; sie enden etwas verjüngt nahe dem Schildchen; letzteres ist gelb, wenig verdunkelt, ebensowenig wie der Hinterrücken, welcher nur einen dunkleren, länglichen Mittelflecken zeigt; derselbe . erreicht das Ende des Hinterrückens nicht und erscheint auch nur bei vorderer Ansicht ganz dunkel. Brustseiten ohne Flecken. Hinterleib gelb, mit breiter, aus grossen, dreieckigen Flecken gebildeter Mittelstrieme und schmalen, schwarzen Seitenstriemen. Die drei letzten Ringe sind fast ganz schwarz, Legeröhre fast gerade, nur etwas nach unten umgebogen, rothgelb.

Hinterbeine dunkelbraun, Hüften und Schenkel, ausser der Spitze, gelb.

Flügel etwas bräunlich, das Stigma blassbraun; Discoidalzelle klein, die obere der aus derselben entspringenden Adern ist mehr als doppelt so lang wie die Zelle.

Schwinger bräunlich.

Körperlänge $15 \mathrm{~mm}$., Flügellänge $13 \mathrm{~mm}$.

Pachyrrhina bo m bayensis Macq. dürfte nahe verwandt sein. Nach Macquart's Angaben sind bei dieser Art die 3 ersten Fühlerglieder gelb und es findet sich am Hinterrand der Hinterleibssegmente je ein schwarzer Flecken. Jedenfalls ist auch das von Samarang stammende Exemplar, welches van der Wulp (Tijdschrift voor Entomol. XXIII, p. 158) als P. bombayensis bestimmte, von den vorliegenden Exemplaren verschieden. Nach seinen eigenen Angaben weichen auch die bezüglichen Exemplare der Sumatra-Expedition nicht unerbeblich ab, sodass die Frage nahe liegt, ob hier nicht vielleicht verschiedene einander ähnliche Arten im Spiele sind.

Bei P. javana fehlt der dunklere Mittelstreifen am Abdomen und die Seitenstriemen sind blasser. Auch soll das Schildchen bräunlich-schwarz sein und die Thoraxseiten schwarze Linien an den Nähten zeigen. Doch ist von dieser Art nur das Männchen beschrieben worden.

Dicranomyia Kobusin. sp. Pasuruan (Java), 3 Exx., Kobus leg. - Fig. 5, 6.

Kopf gelblich, Hinterkopf weisslich, Fühler schwarzbraun, 14-gliedrig.

Thorax vorn ziemlich stark vortretend, oben mattbraun, mit dunklerem Saume, von welchem sich die weisse Farbe der Brustseiten scharf abhebt. Hinterrücken braun, weisslich schimmernd.

Hinterleib braun, mit helleren Hinterrändern, unten lichter, nur die Vorderränder der Segmente verdunkelt. Haltzangen des $\sigma^{\top}$ fast gerade, überall gleichbreit, am Ende stumpf, relativ klein. Legeröhre des $\&$ kurz, gerade.

Beine lang und dünn; Schenkel braungelb, mit dunklerer Spitze; Schienen weiss, in der Mitte mit dunklem Ring. Tarsen weiss; die Schienen und Tarsen sehr kurz, aber dicht weiss behaart; Krallen an der Wurzel mit dem gewöhnlichen Höcker, ganz ohne Zähne; Haftläppchen rudimentär.

Flügel lang und schmal, fast glashell, mit rundem, dunkelbraunem Stigma. Hilfsader etwas jenseits des Ursprungs der Radialader ausmündend. Discoidalzelle unten offen.

Körper- und Flügellänge $7 \mathrm{~mm}$.

Ebenso schmale Flügel, wie bei der vorliegenden Art, finden sich auch bei D. longipennis Schumm. Hier ist die Discoidalzelle jedoch oben offen; nach Osten Sacken kommt eine unten offene Discoidalzelle in dieser Gattung nur bei D. pubipennis O. S. vor; meistens ist sie jedoch auch bei dieser Art geschlossen.

Ebensolche zarte, weisse Tarsen, welche an die von Dolichopeza erinnern, wurden auch 
bei anderen süd-asiatischen Tipuliden angetroffen, so bei Dicranomyia saltans Dolesch. und einigen Mongoma-Arten. Von ersterer ist die vorliegende Art durch das vorhandene Flügelstigma und, vielleicht jedoch nicht immer, durch die offene Discoidalzelle zu unterscheiden; M ongo ma hat ein ganz anderes Flügelgeäder.

Rhipidia pulchra n. sp. Tosari (Java), 1 9 , Kobus leg. - Fig. 7.

Stirne und Rüssel bräunlich-grau; Fühler gelblich-weiss, ziemlich stark behaart, aber ohne kammförmige Anhänge, die unteren Glieder des Schaftes auffällig breiter als die folgenden. Thorax oben sammetartig dunkel kastanienbraun; auch das Schildchen und der Hinterrücken von dieser Farbe. Brustseiten weiss, mit einer breiten, fast sammetschwarzen, vom Halse bis zur Hinterleibsbasis verlaufenden Strieme. Derselben parallel verläuft etwas mehr nach unten eine ebensolche schmälere, theils über die Thoraxseite, theils über die Hüften. Hinterleib braun, der 1e Ring von der Farbe des Thoraxrückens; ferner an jeder Seite ein fast schwarzer, sich nach hinten verjüngender Längsstreifen. Legeröhre bräunlich-gelb, an der Spitze etwas aufgebogen. Flügel mit zahlreichen kleinen, wenig hervortretenden Fleckchen und überdies mit einigen grösseren von viel dunklerer Farbe; das grösste an der Wurzel der Cubitalader, ferner kleinere an den Enden der Hilfs-, Subcostal- und Radialader, ein Fleckchen in der Mitte der Subcostalader, ein solches nahe dem. Ende der Axillarader, eines am Ende der Discoidalader und eines am unteren Ende der hinteren Querader. Auch die marginale QQuerader ist braungesäumt. Schwinger, weisslich. Es ist nuir ein Mittelbein übrig geblieben; dasselbe ist gelblich-weiss mit dunkler Schenkel- und Schienenspitze und dunklem Tarsus.

Körperlänge $6,5 \mathrm{~mm}$; Flügellänge $7 \mathrm{~mm}$.

Die Art zeigt besonders mit Rh. punctiplen a Mik '), aus. Salzburg, sehr grosse Aehnlichkeit, namentlich in der Farbe der Flügel und in den dunklen Striemen an den Thoraxseiten. Als Unterschiede sind folgende hervorzuheben: die 2te Längsader entspringt aus der Iten viel weiter distalwärts, in einer Entfernung von der die beiden Aeste der 1ten Längsader verbindenden Querader, welche kaum grösser ist als diese Querader selbst; in dieser Hinsicht verhält sich die Art also fast wie Rh. maculata. Der Thorax ist oben einfarbig, während Ph. punctiplena daselbst Striemen aufweist. Wenigstens beim vorliegenden Exemplar findet sich kein dunkles Fleckchen ganz nahe der Flügelwurzel, die hintere Querader liegt noch etwas vor der Discoidalzelle, und an den Seiten des Hinterleibes findet sich nur. je eine dunkle Längsstrieme.

Conosia irrorata Wied. Pasuruan (Java), einige Exemplare, Kobus leg.

Die Farbe der vorliegenden Exemplare ist röthlich aschgrau. Am Thorax lassen sich eine wenig verdunkelte Mittellinie und ebensolche breitere, sich nicht bis vorn erstreckende Seitenstriemen erkennen. Vorn am Thorax jederseits ein dunkles, mit den Striemen nicht zusammenhängendes Strichelchen. Zwischen den Striemen kommen zahlreiche, schwarze Punkte vor; ebensolche finden sich auch an den Seitenrändern des Abdomens, an den letzten Ringen auch in der Mitte. Ausserdem trägt der Hinterleib eine schmale, dunkle Mittelstrieme. Die aus der Discoidalzelle hervorgehende Gabelzelle ist öfters wenig länger als ihr Stiel.

Eriocera nigripennis n. sp. (van der Wulp descr.). Hili Madjedja, N. Nias, 1 , Kanne. gieter leg.

Schwarz, das Abdomen zum Theil rothbraun; Flügel schwärzlichbraun.

Mit Ausnahme der Wurzelhälfte des Abdomens ist der ganze Körper, einschliesslich der Fühler, Taster, Beine und Schwinger, tiefschwarz. Fühler dünn, langbehaart, fast von doppelter Kopf- 
länge. Rückenseite des Thorax etwas glänzend; die Brustseiten und ein Streifen von den Schultern bis zur Flügelwurzel, desgleichen auch der Kopf, matt grau.

Hinterleib schlank, sammetschwarz, die rothbraune Farbe ist auf die Oberseite der 3. ersten Ringe beschränkt; Legeröhre braun.

Flügel schwarzbraun, längs dem Vorderrande und an der Spitzenhälfte am dunkelsten, die Kerne der Hinterzellen etwas lichter. Von letzteren sind bei dieser Art 5 vorhanden; die Gabel an der Flügelspitze kurz, halb so lang wie der Stiel; Discoidalzelle länger als breit. Körperlänge $16 \mathrm{~mm}$; Flügellänge $16 \mathrm{~mm}$.

Von den übrigen Eriocera-Arten zeichnen sich 3 durch fast oder ganz einfarbig dunkle, schwarze oder braune Flügel aus: E. albonatata Löw von Mozambique und Ceylon, E. plecioides Walk. von Singapore und E. morosa O. S. von Celebes. Erstere hat Fühler und Beine von gelber Farbe und 3 weisse Punkte nahe der Flügelspitze, woher der Artname; es mag bemerkt werden, dass die neue Art von ebensolchen Fleckchen eine sehr geringe Spur zeigt, doch ist die Identität ausgeschlossen, da E. a lb o n o ta ta nur 4 Hinterzellen besitzt.

Bei E. plecioides ist der Thorax blassröthlich; E. morosa, von welcher ebenfalls nur das O bekannt ist! scheint nahe verwandt zu sein, hat aber ein ganz schwarzes Abdomen; dies würde aber kaum ein. Hinderniss sein, das vorliegende Exemplar dieser Art zuzurechnen, indem auch E. albonotata nach Osten Sacken in der Färbung nicht constant ist. Die Beschreibung, welche Osten. Sacken vom Flügelgeäder von E. morosa giebt, triftt aber für dasselbe nicht zu; ob E. morosa zu den Arten mit 4 oder 5 Hinterrandzellen gehört, geht aus derselben nicht hervor.

\section{STRATIOMYIDAE.}

\section{Hermetia fenestrata n. sp. Lahat (Palembang), $1 \sigma^{7}$, Giesberts leg. - Fig. 9.}

Kopf und Rüssel gelb, auf der Stirn ein grosser dunkelbrauner, etwas gewölbter Flecken, welcher die kurzbehaarten Augen fast berührt; neben letzteren findet sich nahe der Fühlereinpflanzung noch jederseits ein dunkles Fleckchen. Fühler dunkelbraun, die Wurzel etwas heller.

Thorax schwarzbraun mit kurzer, weisslicher Behaarung; Schulterbeulen, die Schwiele hinter den Flügeln und der Rand des Schildchens gelb. Die Thoraxseiten schwarzbraun, gerade vor den Flügeln verläuft ein gelber Streifen nach unten hin.

1 ter Hinterleibsring schwarz; 2ter beinahe ganz durchsichtig gelblich-weiss, nur ein mittlerer Streifen und ein schmaler lateraler Saum schwarz; die folgenden Ringe schwarz mit rostgelben, weissschimmernden Hinterrändern, 5ter Ring fast ganz rostgelb, nur ganz vorne an den Seiten verdunkelt.

Bauch gelblich-weiss, 3ter und 4ter Ring ausser an den Rändern schwarzbraun, 5ّter Ring am Vorderrand von derselben Farbe.

Schenkel schwarzbraun, Schienen gelbweiss mit verdunkelter Endhälfte; die der vorderen Beine nur an der Vorderseite etwas dunkler.

Flügel an der Spitzenhälfte etwas verdunkelt, das Stigma schwarzbraun. Schwinger weissgelb. Körperlänge 14 mm.; Flügellänge $12 \mathrm{~mm}$.

Herm'etia laeta n. sp. $2 \sigma^{\top} \sigma^{\top}$ und 3 \% , gezüchtet aus einer Betelnusspalme in Backergunge (Bengalen). Im Indian Museum, Calcutta. - Fig. 8.

$\sigma^{7}$ Untergesicht und Stirne gelb, letztere mit schwarzer Querbinde über der Mitte und davor jederseits neben den Augen mit einem dreieckigen, schwarzen Fleckchen. Ocellenfleck und Hinterseite des Kopfes, sowie die Backen, schwarz. Fühler schwarz mit 2 braungelben Wurzelgliedern.

Thorax schwarz, mit' nur sehr geringem Glanz; über die Mitte verläuft eine wenig auffällige, weisse Längslinie, welche sich bis zur Quernaht erstreckt. Schulterbeulen und ein sich daran nach innen anschliessendes, dreieckiges Fleckchen gelb. Zu beiden Seiten des Thorax verläuft eine Strieme 
von derselben Farbe, welche ebensowenig die Quernaht überschreitet, und vorn schmal mit der Schulterbeule verbunden ist. Schildchen gelb, nur an der äussersten Basis schwarz. Zwischen demselben, und der Flügelwurzel ein gelber Flecken.

Brustseiten schwarz mit zwei gelben Flecken; von diesen liegt ein L. förmiger oberhalb der Mittelhüften; ein kleinerer findet sich oberhalb der Hinterhüften.

Schwinger gelb, der Stiel etwas gebräunt.

Die drei ersten Ringe des Abdomens sind schwarz, die folgenden rothbraun. Am 2 ten Ringe finden sich nahe dem Vorderrande 2 etwas dreieckige, weissliche Fensterflecken neben einander, ferner am Hinterrande jederseits ein gelber Flecken, welcher den schmalen, gelben Seitenrand dieses Ringes berührt; auch die Seitenränder der folgenden Ringe sind schmal gelb. Der $3^{\text {te }}$ und 4te Ring zeigen einen gelben Hinterrand, am $5^{\text {ten }}$ Ringe findet sich hinten ein gelber Mittelflecken, welcher breiter als lang ist. Am Bauche sind die drei ersten Ringe schwarz; der 2te jedoch bis auf einen schmalen, hinteren und seitlichen Theil durchsichtig, der 4 te und 5 te Ring rothbraun; diese Farbe setzt sich auch noch auf das Medianfeld des 3ten Ringes fort, welches ganz vorn sogar gelb erscheint.

Beine gelb, mit Ausnahme der Schenkel, welche an den Hinterbeinen ganz, an Vorder- und Mittelbeinen bis auf die Spitze schwarz sind; die Schienen höchstens am Ende etwas gebräunt.

Flügel an der vorderen Hälfte gelbbraun, an der hinteren Hälfte und aussen mit dunkelbraunem Anfluge.

Körperlänge $13 \mathrm{~mm}$. , Flügellänge $11 \mathrm{~mm}$.

Beim Weibchen sind die beiden Fensterflecken des 2 ten Ringes relativ kleiner und liegen unmittelbar am Vorderrand; auch die seitlichen Flecken sind weniger ausgebildet und weiter von einander getrennt, die beiden letzten Ringe sind nicht rothbraun, sondern schwarz; der Mittelflecken

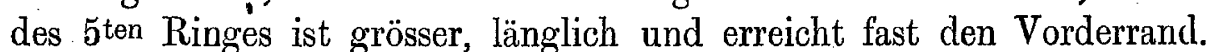

Bei einem Weibchen fehlen die oberhalb der Fühler neben dem Augenrand liegenden schwarzen Fleckchen.

Die Art ist mit H. cerioides Walk. nahe verwandt; ich finde für letztere jedoch keinen Mittelflecken am letzten Segmente angegeben, und derselbe fehlt auch dem mit der Walker'schen Art synonym erklärten batjanen sis v. d. Wulp; bei letzterer sind die Flügel und die Schienen. dunkler gefärbt, letztere beinahe ganz schwarz. Anch ist der Hinterleib des Männchens von $\mathrm{H}$. cerioides Walk. wohl nicht theilweise rothbraun, sonst hätte Osten Sacken, dem auch Männchen zur Verfügung standen, dies wohl besonders hervorgehoben.

Eudmeta brunnea n. sp. Darjeeling (Bengalen) $1 \sigma^{7}, 2$ \%

Kopf gelbbraun, Fühler schwarzbraun.

Thorax gelbbraun, die Rückenseite etwas verdunkelt.

Hinterleib gelblich-braun, vom 2ten Ringe an mit zwei schwarzbraunen Längsbändern nahe dem Seitenrande; am 4ten und $5^{\text {ten }}$ Ringe sind die Ränder fast verschmolzen, sodass die ganze Oberseite schwarzbraun erscheint; nur findet sich am 4ten noch ein viereckiger Mittelflecken von der Grundfarbe; auch ist der Vorderrand dieses Ringes schmal gelb.

Bauch gelblich-braun, die letzten Segmente etwas verdunkelt.

An den Beinen sind die vorderen Tarsen am Ende verdunkelt, die Hinterschienen und -Tarsen schwarz, sonst ist auch hier Alles gelblich-braun.

Flügel etwas verdunkelt, am Vorderrande mehr gelblich, die Spitze und eine Binde über die Mitte schwarzbraun; in der Analzelle ein mit dieser Binde zusammenhängender dunklerer Wisch. Körperlänge 14 mm.; Flügellänge ebenfalls $14 \mathrm{~mm}$.

Beim Weibchen ist der Hinterleib in der Mitte weniger verdunkelt. Der Metatarsus der Hinterbeine ist bei demselben so lang wie die Schiene, während er beim $\sigma^{\nearrow}$ etwas kürzer ist.

Microchrysa flavi yentris Wied. Pasuruan (Java), 107,1 \%, Kobus leg. 
Ptecticus melanurus Walk. Darjeeling, $3 \sigma^{\top} \sigma^{\top}$.

Ich zweifle nicht, dass die vorliegenden Exemplare mit dieser, von Walker zunächst nach einem kopflosen Exemplare als eine Ctenophora beschriebenen Art identisch sind. Schon Osten Sacken hat darauf hingewiesen, dass hier von einem mit Sargus verwandten Thier die Rede ist. Obgleich bei keinem der Exemplare das 3te Fühlerglied noch vorhanden ist, so lässt sich doch der nagelförmige Fortsatz des 2 ten Gliedes gut erkennen, sodass die Art ohne Zweifel in die Gattung Ptecticus eingereiht werden muss.

- Die Art ist fast ganz rothgelb, das Untergesicht und der etwas gewölbte Theil der Stirne, welcher gerade oberhalb der Fühler liegt, weisslich, der Ocellenfleck schwarz; die 3 dunkleren Längsstriemen des Thoraxrückens sind immer wenig deutlich, bisweilen kaum wahrnehmbar. Am Hinterleib sind die 4 ersten Ringe gelb, der Rest schwarz, bei einem Exemplar ist auch der $3^{\text {te }}$ und 4te Ring oben zum Theil verdunkelt. Die Flügel sind honiggelb, mit schwarzbrauner Spitze; letztere ist durch eine fast gerade Linie, welche etwas jenseits der Discoidalzelle liegt, von der gelben Partie abgetrennt. Bei 2 Exemplaren ist auch der Hinterrand der Flügel mehr oder weniger verdunkelt.

Die Hintertarsen sind bisweilen schwärzlich, bisweilen aber auch lichter, sodass z. B. nur der Metatarsus an der Oberseite geschwärzt erscheint.

Die Grösse variirt zwischen 13 und $18 \mathrm{~mm}$., die Flügel sind so lang wie der Körper.

Ptecticus tricolor n. sp. (v. d. Wulp descr.) Sukabumi, W. Java, $1 \sigma^{7}$, Fruhstorfer leg. Fig. 11.

Thorax schwarz mit gelben Längslinien; Hinterleib roth, der $1^{\text {te }}$ und die beiden letzten Ringe schwarz; Fühler bräunlich-gelb; Beine gelb, das Ende der Schenkel und an den Hinterbeinen auch die Wurzel derselben, die Schienen und Tarsen schwarz.

Kopf relativ gross; die Stirue in der Mitte so schmal, dass sich die Augen fast berühren, nach dem Scheitel hin allmählich etwas verbreitert und glänzend schwarz, ganz hinten mit feiner, schwarzer Behaarung; der oberhalb der Fühler liegende Theil der Stirne bildet ein stark gewölbtes Dreieck von weisslicher Farbe. Das sehr kurze Untergesicht ist weisslich-gelb mit gelber Behaarung. Fühler bräunlich, die 2 ersten Glieder dunkler, etwas glänzend, wodurch der nagelförmige Fortsatz des 2 ten Gliedes leicht erkennbar ist, das 3te Glied heller, matt, breit eiförmig, mit schwarzer Rückenborste. Rüssel rothgelb.

Thorax nach vorne etwas verschmälert, mattschwarz, der Hals, die eckig vorspringenden Schulterbeulen, ein bẹi denselben anfangender und bis zu der Flügelwurzel verlaufender Streifen und 2 ganz durchlaufende Längslinien am Thoraxrücken gelb; an der Brustseite ein grosser weisser Flecken oberhalb der Mittelhüften, auch die Seiten des Hinterrückens von dieser Farbe; letzterer mit ziemlich langer, weisser Behaarung. Schildchen schwarz mit namentlich an den Seiten breitem, gelbem Rande.

Hinterleib an der Basis etwas verengt, am 4ten Ringe am breitesten, der 1te Ring schwarz, an beiden Seiten weiss und mit langer, weisser Behaarung; der 2te, $3^{\text {te }}$ und 4 te, sowie der Vorderrand des $5^{\text {ten }}$ Ringes roth, letzterer Ring im Übrigen schwarz und nach hinten verengt; der 6te Ring ebenfalls schwarz, kleiner und schmäler als die vorhergehenden und hinten spitz.

Beine blass rothgelb; Vorderhüften gelb, die übrigen schwarz, an den Mittel- und Hinterbeinen díe Spitzenhälfte der Schenkel, an den Hinterbeinen auch die Wurzel derselben, die Schienen und Tarsen schwarz, auch die letzten Glieder der Vorder- und Mitteltarsen, sowie die Spitze der Vorderschienen etwas verdunkelt. Flügel bräunlich, an der Wurzel und am Anfang des Hinterrandes fast hyalin, am Fnde des Vorderrandes etwas dunkler, das Stigma kaum angedeutet; die Adern braun, an der Basis gelblich. Schwinger rothgelb.

Körperlänge $18 \mathrm{~mm}$; ; Flügellänge $18 \mathrm{~mm}$.

Die Art hat die für das Genus Ptecticus charakteristische Fühlerform, was nicht gerade 
von allen zu demselben gestellten Arten gesagt werden kann, indem manche nur wegen der zum Theil rothgelben Körperfarbe in dasselbe untergebracht wurden. Die gelbe Zeichnung des Thorax unterscheidet sie von allen anderen.

Nach van der Wulp's handschriftlicher Angabe findet sich ein zweites Männchen im Brüsseler Museum; dasselbe ist aber bedeutend kleiner.

Negritomyia sp. Darjeeling (Bengalen), 1 Ex.

Schwarz, etwas glänzend. Fühler ebenfalls schwarz, oberhalb derselben zwischen den kurzbehaarten Augen jederseits ein weisses Fleckchen, welches von weissen Härchen gebildet wird.

Kopf und 'Thorax sehr kurz weiss behaart; an den Seiten des letzteren ist die Behaarung etwas länger. Schulterbeulen mit gelbem Punkt; Dornen des Schildchen schwarz, an der alleräussersten Spitze gelblich.

Hinterleib grösstentheils mit dunkler Behaarung; ein weisser Flecken am Seitenrand und ein dreieckiger in der Mitte der Ringe weiss behaart. Der Mittelfleck des letzten Ringes ist länglich rechteckig.

Bauchseite glänzend schwarz, kurz weiss behaart. Beine fehlen dem Exemplare.

Flügel zum grössten. Theil verdunkelt, an der Spitzenhälfte eine breite, dunkelbraune Binde, welche auch die äussere Hälfte der Discoidalzelle und fast die ganze Analzelle ausfüllt. Flügelstigma dunkelbraun.

Körperlänge $12 \mathrm{~mm} .$, Flügellänge $11 \mathrm{~mm}$.

Das Exemplar ist mit N. maculipen nis Macq. nahe verwandt; bei letaterer sind aber die Dornen des Schildchens grösstentheils gelbbraun; beim obigen Exemplare findet sich auch am $3^{\text {ten }}$. Ringe ein weisser Mittelflecken und die Flügel sind über einen viel grösseren Theil verdunkelt.

Auch N. albitarsis aus Neu-Guinea stimmt wohl sehr mit obigem Exemplare überein; das $3^{\text {te }}$ Fühlerglied ist aber an der Innenseite lichter; auch sind die Dornen des Schildchens bei meinem Exemplare so gut wie ganz schwarz.

Weil das Exemplar zu schlecht conservirt ist, unterlasse ich es dasselbe zu benennen, und möchte bloss auf das Vorkommen der Gattung in Vorder-Indien hinweisen.

Ptilocera quadridentata F. Pasuruan (Java), 1 \%, Kobus leg. - Fig. 12.

Die Schuppen, welche die gelbe Zeichnung bei dieser Art veranlassen, haben die in Fig. 12 abgebildete Gestalt. Rippen zeigen sich an denselben nicht, sie sind ganz glatt.

Tinda ind ica Walk. Pasuruan (Java), $10^{\top}$, Schat leg.; 1 ㅇ. Kobus leg.

Rosapha bimaculata n. sp. (v. d. Wulp descr.) Gunong Tji Salimar, W. Preanger, Java, i ठৃ, Kannegieter leg. - Fig. 13, 14.

Rothgelb, die Hinterschienen und die Vordertarsen schwarz, Mittel- und Hintertarsen weiss, nur am Ende schwarz. Flügel mit 2 braunen Flecken.

Augen in langer Strecke zusammenstossend, sodass unten die Stirne fast ganz verschwindet, der Ocellenhöcker sehr vorgezogen. Stirne schwarz. Untergesicht kurz, schwarz, neben den Augen mit schmalem, weissem Saume. Fühler länger als der Kopf, in der Profilansicht an die Unterseite desselben gerückt, röthlich gelb, der lange Endgriffel schwarz, fadenförmig, etwas abgeflacht, beiderseits äusserst kurz und dicht behaart. Rüssel schwarz.

Thorax, Schildchen und Hinterleib rothgelb, wenig glänzend; der Thorax länger als breit, nach vorn nưr wenig verschmälert, in der Mitte des Rückens ein brauner Längsstreifen, welcher die Quernaht nicht überschreitet, an den Seiten zwischen den Schultern und der Flügelwurzel eine hellgelbe Längslinie. Brust schwärzlich. Schildchen gross, hinten abgestutzt, mit einem bräunlichen Längsistreifen und 4 heller gelb gefärbten Dornen, von welchen die beiden mittleren bedeutend grösser sind als die äusseren.

Hinterleib elliptisch. 
Beine rothgelb; Hinterschienen, sowie die Vordertarsen schwarz; die Mitteltarsen sind in ${ }^{2} / 3$ ihrer Länge (das $1^{\text {te }}$ und 2te Glied), die Hintertarsen zur Hälfte (nur der Metatarsus) weiss.

Flügel hyalin, der Randfleck dunkelbraun; am Ende des Vorderrandes findet sich ein zweiter dunkelbrauner Flecken, welcher sich nach unten, obgleich verwaschen, noch etwas über die lte der 3 aus der Discoidalzelle entspringenden Adern erstreckt. Die kleine Querader sehr kurz. Schwinger gelb.

Körperlänge $6 \mathrm{~mm}$; Flügellänge $6 \mathrm{~mm}$.

Die Art scheint der R. (Calochaetis) bicolor Big. (Ann. Soc. Ent. France 1879. p. 189) nahe verwandt, jedoch ist bei dieser der Hinterleib in der Mitte schwarz; auch ist der Rüssel braungelb und der Schwingerknopf ist braun.

\section{LEPTIDAE.}

Chryso pila simplex n. sp. Tosari (Java), 5 Exx., sämmtlich Männchen, Kobus leg. - Fig. 15.

Augen des $\sigma^{\top}$ fast zusammenstossend, nur durch eine feine schwarze Linie getrennt, welche vorn in das schwarze Stirndreieck übergeht. Fühler gelbbraun oder braun, das 3te Glied kurz und rund, mit Endborste. Ocellenhöcker stark vortretend. Untergesicht vertieft.

Thorax mattbraun, mit 4 fast zusammenfliessienden, dunkleren Längsstriemen, von welchen die mittleren schmäler sind als die äusseren. Brustseiten aschgrau. Schildchen gelb, an der Wurzel etwas verdunkelt. Hinterleib von derselben Farbe wie der Thorax, der 2te, 3te und 4te Ring desselben heller als die übrigen, und mehr oder, weniger durchscheinend und gelblich. Beine ganz gelbbraun.

Flügel etwas gebräunt, das Randmal dunkler braun, nicht scharf begrenzt. Discoidalzelle von der in Fig. 15 abgebildeten Gestalt. Der obere Gabelarm der Radialader zeigt bei einigen Exemplaren nahe der Basis ein bräunliches Fleckehen, in welchem sogar bisweilen ein kurzer Aderanhang sichtbar ist. Bei einem Exemplare findet sich an der bezüglichen Stelle des rechten Flügels ein bis zur Subcostalader durchlaufendes Aderchen, während der linke daselbst nur einen kurzen Aderanhang zeigt.

Schwinger gelblich mit dunkelbraunem Knopfe. Körperlänge $6 \mathrm{~mm}$. Länge der Flügel ebenfalls $6 \mathrm{~mm}$.

Die Art sieht, was die Farbe anlangt, der ${ }^{-} \mathrm{C} h \mathrm{r}$. ferrugin os a ähnlich, unterscheidet sich aber durch geringere Grösse, durch das vertiefte Untergesicht, das mehr hervortretende Flügelstigma und den nicht gelb. gefärbten Flügelvorderrand.

\section{BOMBYLIIDAE.}

Argyromoeba distigma Wied. Darjeeling 1 Ex.; Pasuruan (Java), Kobus leg.

Die Art hat ein weites. Verbreitungsgebiet, denn Coquillett ${ }^{1}$ ) giebt sie auch aus Japan an.

\section{SYRPHIDAE.}

Ceria trinotata n. sp. Darjeeling, $1 \sigma^{7}$.

Schwarz mit schwefelgelber Zeichnung, der Ceria javana Wied. ähnlich.

Kopf glänzend gelb, Scheitel sowie der hintere Augenrand gelb, mjt schwarzer Längsstrieme, in welche hinten die Ocellen gelagert sind. Zu beiden Seiten des Scheitels ist der Augenrand eine

1) Coquillett. Report on a collection of Japaneșe Diptera. Proc. U. S. Nation. Museum XXI. 1898. p. 318. 
kurze Strecke weit verdunkelt. Auch die kurze Stime hat eine schwarze Mittelstrieme; dieselbe erweitert sich näch vorn etwas und trägt daselbst den Fühlerstiel.

Untergesicht gelb mit glänzend schwarzer Mittelstrieme; die gelbe Farbe steht mit derjenigen der Stirne in offener Verbindung. Backen schwarz. Hinterkopf schwarz, gerade am unteren Rande mit schmalem, gelbem Flecken, welcher auch das Auge berührt. Fühler schwarz, das 3te Glied sehr dunkelbraun, der Griffel weiss. Die 3 Glieder fast von gleicher Länge, zusammen etwa so lang wie der Fühlerstiel. Thorax und Hinterleib schwarz, wenig glänzend, mit sehr kurzer brauner Behaarung. Schulterbeulen und ein dahinter gerade vor der Quernaht liegender, kleinerer Flecken, die Seitenränder hinter der Quernaht fast bis zum Schildchen und ein kürzerer Flecken median zwischen diesen beiden Striemen gelb. Der zuletat genannte ist längsoval und erreicht weder die Quernaht noch das Schildchen. Pleuren glänzend schwarz, mit 3 nur durch schmale, schwarze Linien getrennten Flecken, je einem auf Meso-, Sterno- und Pteropleurum. Schildchen schwarz mit gelbem Rande.

Hinterleib kolbenförmig, an der Wurzelhälfte sehr schmal, namentlich der 2te Ring, der so lang ist wie die 3 folgenden Ringe zusammen.

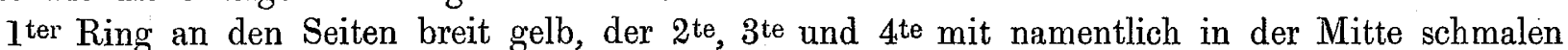
gelben Hinterrandsäumen. Bauch schwarz, nur der 2te Ring ganz hinten gelb. Beine schwarz, die Schienen und Tarsen mit sehr kurzer brauner Behaarung. Schenkel an der Hinterseite mit einem grossen, länglichen, gelben Flecken; an den Hinterschenkeln findet sich derselbe auch vorn, indem er nur an der Oberseite einen schmalen, schwarzen Längsstreifen übrig lässt. Schienen und die Wurzel der Hintertarsen bräunlich, erstere in der Mitte schwarz.

Flügel namentlich an der Vorderrandshälfte mit bräunlichem Anfluge, auch die 5te Längsader braun gesäumt. Discoidalzelle fussförmig, die dieselbe oben begrenzende Ader in der Mitte eingebuchtet, bogig, aber ganz ohne Aderanhang.

Schüppchen weiss. Schwinger röthlich-gelb.

Körperlänge $21 \cdot \mathrm{mm}$; Flügellänge $19 \mathrm{~mm}$.

Die Art ähnelt sehr der C. javana Wied., ist aber bestimmt verschieden. Bei letzterer sind das Scheiteldreieck und die Backen ganz schwarz, das Gelb der Stirne ist durch ein schwarzes Querbändchen von dem des Untergesichtes abgetrennt. An den Fühlern übertrifft das 2te Glied das 1 te etwas und das 3 te bedeutend àn Länge, die Borste ist länger und dünner. Am Thorax fehlen die 3 Längsstriemen hinter der Quernaht und das Schildchen ist nur an den Seiten gelb. Der 2te Hinterleibsring ist relativ noch schmäler und länger; die gelben Hinterrandssäume sind namentlich an den Seiten schmäler und erreichen den Seitenrand nicht. In der Farbe der Beine stimmen die beiden Arten fast überein, bei C. javanica findet sich jedoch an der Unterseite der Hinterschenkel ein schwarzer Längsstreifen. Die Flügel sind bis zur 3 ten Längsader schwarzbraun, viel dunkler als bei C. trinotata, die Hinterrandshälfte hingegen glashell. Die Discoidalzelle hat bei beiden Arten dieselbe Form, ist also auch bei C. javanica oben nur eingebuchtet. Letztere Art ist im Ganzen etwas kleiner (Körperlänge $20 \mathrm{~mm}$., Flügellänge $13 \mathrm{~mm}$ ).

Microdon metallicus n. sp. Darjèling (Bengalen), 1- $\sigma^{7}$.

Glänzend stahlblau, die Oberseite des Thorax mehr violet; Oberseite des Körpers dicht punktirt. Fühler mattschwarz, das erste Glied so lang wie das 2 te und 3te zusammen, das 2te nur ein Viertel von der Länge des 3ten erreichend. Untergesicht mit dichter, weisser Behaarung. Augen schwarzbraun, die Facetten überall gleichgross. Thorax oben sehr kurz schwarz behaart, am Schildchen mehrere lichte Haare und die Dornen des Schildchens weiss behaart. Brustseiten fast nackt. Hinterleib mit sehr kurzer, dunkler Behaarung, vorn an den Seiten der Segmente weisse Haarflecken, ferner oben je 2 ebensolche nahe dem Hinterrande des $3^{\text {ten }}$ und 4 ten Ringes; namentlich die des letzteren sind von bedeutender Grösse. Zwischen dem 3 ten und 4ten, und dicht vor dem Ende des $4^{\text {ten }}$ Ringes finden sich je.2 Quereindrücke. Beine schwarz.; Schienen an der Aussenseite dicht kurz weissbehaart.

Flügel glashell.

Körperlänge $11 \mathrm{~mm}$; Flügellänge $9 \mathrm{~mm}$. 
Durch die metallische Körperfarbe erinnert diese Art an Microdon stilboides Walk. Letzterer ist aber glänzend grün, und ferner ist in Walker's Beschreibung weder von weissen Haarflecken am Hinterleib, noch von weisser Behaarung an den Beinen die Rede. Auch sollen die Flügel gebräunt sein.

Paragus serratus F. Pasuruan (Java), $1 \sigma^{\pi}$, Kobus leg.

Das Exemplar stimmt gut mit Schiner's Beschreibung; nur ist die schwarze Strieme des Untergesichtes wenig deutlich.

Eunerus aurifrons Wied. Pasuruan (Java), 1 Ex., Kobus leg.

Das vorliegende Exemplar hat etwas ausgebreitetere gelbe Färbung an den Beinen als es von Wiedemann angegeben wird. Das Ende der Schenkel und die Schienen sind von dieser Farbe, letztere mit brannem Ringe vor der Spitze.

Milesia gigas Macq. West-Sumatra. Im Leydener Museum.

Milesia semifulva n. sp. Darjeeling (Bengalen) $1 \sigma^{\top}$. - Fig. 16.

Kopf gelblich-weiss mit einigem weisslichen Schimmer. Fühler rothgelb. Der Mundrand springt, wie bei M. Reinwardti, ziemlich stark vor. Scheitel gelblich-braun.

Der Thorax oben grösstentheils schwarzbraun, die Schulterbeulen und ein in der Mitte unterbrochener, schmaler, nicht scharf begrenzter, über die Quernaht verlaufender Streifen rothbraun. Die Seiten des Prothorax und die Umgebung der Flügelwurzel rothbraun. Schildchen ganz schwarz. Am Hinterleib ist der lte Ring schwarzbraun; der zweite gleichgefärbt, aber mit einem durchsichtig gelben, sich nach den Seiten erweiternden Vordersaume. Der 3te und 4te Ring sind grösstentheils rothgelb, am Vorderrande etwas lichter, besonders in der Nähe des Hinterrandes mit röthlichem Anflug.

Am Bauche ist der 1te Ring an den Seiten, der 2te fast ganz durchsichtig, weisslich, letzterer mit einem grossen, dreieckigen, vorn mit seiner Spitze den l en Ring nicht erreichenden, bräunlichen Mittelflecken, die folgenden Ringe sind daselbst braun, mit breiten, weisslichen Einschnitten.

Beine rothbraun, Vorder- und Mittelschenkel unten, Hinterschenkel fast ganz schwarz, unten nahe der. Spitze mit einem Dorn. An den Schienen aussen ein schmaler, schwarzer Streifen. Flügel namentlich am Vorderrande bräunlich-gelb; vor der Spitze ein wenig auffälliger, dunklerer Schatten.

Schwinger gelbbraun mit rothbraunem Knopfe.

Körperlänge $25 \mathrm{~cm}$.; Flügellänge $18 \mathrm{~cm}$., also von der Grösse von M. Reinwardti.

Die Art ist besonders mit M. gigas Macq. nahe verwandt, auf welche auch Osten Sacken's Bestimmungstabelle führt; die Färbung des Hinterleibes ist aber eine andere.

Helophilus curvigaster Macq. Macquart. Dipt. exot. II. 2. 62. Taf. XI. Fig, 1.

Darjeeling (Bengalen) $1 \sigma^{\top}$; in meiner Sammlung ein $ᄋ$ unbekannter Herkunft.

Weil Macquart's Beschreibung sehr kurz gehalten ist, gebe ich hier folgende Erweiterung:

$\sigma^{7}$. Augen nackt, in einem Punkt zusammenstossend. Scheitel mattschwarz, schwarz behaart; Stirne und Untergesicht dicht weiss behaart mit schmalem, glänzend schwarzem Mittelstreifen, welcher die Fühler bei weitem nicht erreicht. Letztere schwarz, mit gelblicher Borste. Nur unmittelbar oberhalb der Finpflanzung der Fühler ist die Stirne glänzend schwarz.

Thorax oben schwarzbraun mit ziemlich dichter, aber kurzer, gelblichweisser Behaarung. Schildchen braunroth. 
Hinterleib nach hinten auffallend verjüngt, am Ende etwas kolbenartig. Am 2 ten Ringe ein braunes und dahinter ein schwarzes Querband, nahe dem Hinterrande. Dasselbe ist in der Mitte am breitesten und erreicht den Seitenrand nicht. Die beiden folgenden Ringe zeigen je 2 mattschwarze Querbänder, welche den Seitenrand erreichen und am 4ten Ringe sogar daselbst unter einander zusammenhängen. Der $5^{\text {te }}$ Ring ist nur am Ende und an den Seiten schwarz. Die rothbraunen Theile des Abdomens sind etwas vertieft, glänzend, die schwarzen Theile matt. Die Einschnitte, sind weisslich. Der ganze Hinterleib ist äusserst kurz behaart, die Härchen meist von weisslicher Farbe, an den schwarzen Partien aber schwarz.

Die vorderen Beine. sind grösstenteils gelbbraun, die Vorderseite und von den Schenkeln auch die Oberseite schwarzbraun, die Hinterschenkel gelbbraun, oben und an der Spitze schwarz, ausserdem mit einem schwarzen Querringe über der Mitte und einem schwarzen Fleckchen an der Aussenseite nahe der Wurzel. Die Schienen sind fast ganz dunkel, die Tarsen gelbbraun. An der Wurzel der Hinterschenkel findet sich ein grosser, stumpfer Höcker von gelbbrauner Farbe. Flügel glashell; am Vorderrande zwischen Hilfsader und Subcostalader ein schwarzes Strichelchen.

Körperlänge $11 \mathrm{~mm}$; Flügellänge $8 \mathrm{~mm}$.

Beim $q$ sind die Augen getrennt, Stirn und Scheitel mit langer, brauner Behaarung, in der Nähe der Fühler ist dieselbe lichter. Zwischen Stirn und Scheitel, da, wo die Augen einander am nächsten liegen, ein glänzend schwarzbraunes, nacktes Querbändchen.

Stirne nach hinten etwas erweitert. Am Untergesicht fehlt der schwarze Medianstreifen; derselbe ist jedoch auch beim $\sigma^{\top}$ nur wenig ausgebildet. Die weisse Querbinde über die Thorakalnaht ist, ebenso wie bei meinem Männchen, wenig deutlich. Der 2te Hinterleibsring zeigt 2 schwarze Querbänder.

Am 3 ten und 4ten Hinterleibsringe sind die schwarzen Bänder breit, sodass sie kaum noch getrennt sind; das daselbst bei dem $\sigma^{7}$ vorhandene rothbraune Querband wird hier fast nur noch durch lichtere Behaarung angedeutet.

An den Hinterschenkeln findet sich kein Höcker.

Körperlänge $14 \mathrm{~mm}$; Flügellänge $11 \mathrm{~mm}$.

Die Beschreibung Macquart's bezieht sich nach den getrennten Augen auf das Weibchen.

Megaspis crassus. F. Darjeeling, $2 . \sigma^{7} \sigma^{7}$.

Die Art sieht M. sculptatus v. d. Wulp sehr ähnlich; bei letzterem fehlen aber die weissen Haarflecken am Rücken und die eingedrückten Ringe am $3^{\text {ten }}$ und $4^{\text {ten }}$ Hinterleibssegmente. Bei M. crassus sind auch die vorderen Schenkel fast ganz roth; der Flecken auf der kleinen Querader hängt, wenigstens bei den mir vorliegenden Exemplaren nicht mit der Bräunung der Flügelwurzel zusammen.

Megaspis zonalis F. Darjeeling, 6 우 ; Borneo, am oberen 'Theile des Sibau-Flusses, Büttikofer leg:

Die Exemplare von Darjeeling sehen den javanischen ganz ähnlich. Dagegen zeigen die von Borneo breitere, schwarze Bänder am Hinterleib; auch sind die Flügel in ausgedehnterer Weise geschwärzt, indem fast die ganze Vorderrandshälfte bis zum Stigma schwarz ist. Dasselbe ist bei einem. Exemplare der Sumatra-Expedition der Fall. Diese stimmen also mit den aus China stammenden Typen von Wiedemann, während bei denen von Darjeeling das Gelb an 3ten und 4 ten Hinterleibssegmente sich viel mehr ausbreitet und selbst Querbänder von dieser Farbe gebildet werden, wie bei den javanischen Exemplaren.

Axona chalcopygus Wied. Sumatra; Ardjoeno (Java), Hekmeyer leg. Im Leydener Museum.

Eristalis argy rocephalus Macq. Ambarawa (Java), Ludeking leg. 1 ㅇ. Desgleichen.

Eristalis nitidus v. d. Wulp. Sumatra. Desgleichen. 
Eristalis splendens Le Guill. Flores, Semmelink leg; Sümatra, Ludeking leg. Im Leydéner Museum.

Volucella ursina n. sp. Darjeeling. 1 .

Kopf glänzend schwarz, auch das Untergesicht; nur an den Seiten desselben ein vom Augenrande fast bis zur Spitze des Untergesichts verlaufender, sich allmählich verjüngender, gelber 8treifen. Auch der Mundrand selbst schmal gelblich. Stirn schwarzbehaart. An den Fühlern sind die beiden Wurzelglieder röthlich-gelb, das nierenförmige dritte Gilied schwarzbraun.

Thorax gelbbraun, am Rücken etwas verdunkelt, mit dichter, ziemlich langer, gelbbrauner Behaarung. Schildchen honiggelb.

Hinterleib schwarzbraun, nur der sehr kurze 1te Ring gelb, mit kurzer, aber dichter, gelber und schwarzer Behaarung; am 2ten Ringe herrscht, namentlich nahe dem Hinterrande, die gelbe Behaarung vor, am 3ten Ringe die schwarze: hier ist nur ein $\Lambda$-förmiges, schmales Querband und der Hinterrand gelb behaart. Der 4te Ring ist fast ganz schwarz behaart, nur vorn an den Seiten finden sich gelbe Härchen. Bauch glänzend schwarz.

Beine schwarz, die Hüften gelb, sowie auch die Schenkel der Vorder- und Mittelbeine mit Ausnahme eines scharf begrenzten, schwarzen Längsstreifens an der Innenseite der Endhälfte. Alle Kniee gelb.

Flügel an der Spitze etwas verdunkelt und mit einem von der Mitte des Vorderrandes entspringenden, schwarzen Flecken, welcher sich ziemlich weit wurzelwärts erstreckt, die Unterrandzelle aber frei lässt und unten nicht über die vierte Längsader, distalwärts nicht über die kleine Querader hinausgeht.

Körperlänge $17 \mathrm{~mm}$; Flügellänge $16 \mathrm{~mm}$.

Die Art ähnelt im Habitus sehr der V. trifasciata Wied., hat aber eine sehr verschiedene Farbe. Sie scheint auch mit V. nubeculos a Big. aus China(?) und jedd on a Big. ${ }^{1}$ ) aus Japan nahe verwandt. Bei ersterer sind aber Stirne, Untergesicht und Antennen rothgelb, sowie auch die Beine, und der Hinterleib hat gelbe Einschnitte. In der Beschreibung von V.jeddona stimmt die lateinische Diagnose nicht mit der französischen überein, was die Geschlechter anlangt. Bei dem einen Geschlecht, welches noch am meisten mit meinem Exemplare übereinstimmt, ist der Hinterleib an den Seiten des $]$ ten und 2 ten Ringes gelb. Wenn hier bei oberer Ansicht sichtbare Seitenflecken gemeint sind, so stimmt dies für mein Exemplar nicht zu; bei diesem ist dies nur ganz an den Seiten der Fall. Auch sind die Beine nicht schwärzlich, sondern zum Theil gelb.

Rhingia cincta n. sp. Tosari (Java), 1 \%, Kobus leg.

Unserer Rh. rostrata ähnlich, aber etwas grösser, Grundfarbe glänzend gelbbraun, Thoraxoberseite und Hinterleib zum Theil dunkler.

Stirne schwarzbraun, glänzend, Untergesicht rothgelb bis gelbbraun. Fühler rothgelb.

'Thorax oben schwarzbraun, mit 2 nur vorn deutlichen, weisslichen Striemen, Schildchen gelbbraun, Brustseiten von derselben Farbe, die Unterseite zwischen Vorder- und Mittelhüften schwärzlich.

Am Hinterleib ist der 1 te Ring gelbbraun, der 2 te hat einen schwarzen Hinterrand, der $3^{\text {te }}$ und 4te Ring schwarz mit schmaler, gelber Querbinde, welche beim 4ten Ringe in der Mitte sehr breit unterbrochen ist. Der 5 te Ring ist ganz schwarz. Bauch braungelb. Beine braungelb; Vorderund Hintërtarsen ganz, Mitteltarsen am Ende verdunkelt. Hinterschienen mit 2 wenig deutlichen, dunklen Querringen. Metatarsen der Mittelbeine relativ schmal.

Flügel gelblich, das Stigma braungelb; die Adern dunkel, die vena. spuria jedoch gelblich.

Körperlänge (das vorspringende Untergesicht nicht mitgerechnet) $9 \mathrm{~mm}$.; Flügellänge $9,5 \mathrm{~mm}$.

Ausser durch die schon oben angegebenen Merkmale unterscheidet sich die Art noch von $\mathrm{Rh}$.

1) Bigot. Diptëres nouveaux ou peu connus, VI. Ann. Soc. Entomol. France. (5) V. $1875,-472$. 
rostrata L. durch das. relativ etwas mehr verlängerte Untergesicht; durch die namentlich am Hinterleibe kürzere Behaarung, sowie auch durch die Farbe, indem bei Rh. rostrata der ganze Thorax dunkel ist, der Hinterleib aber mehr Gelbes zeigt.

Auch die amerikanische $R$ h. nasica Say sieht der Rh. rostrata ähnlich. Sie unterscheidet sich von der indischen Art durch die Längsstrieme des Hinterleibes, durch das blassgelbe, jederseits ein braunes Fleckchen aufweisende Schildchen, durch ganz dunklen Thorax und durch anders gefärbte Beine.

Baccha pulchrifrons Austen. Java, 1 ○, Piepers leg.

Baccha refulgens Austen. Austen. New Syrphidae in the Collection of the British Museum. Proc. Zool. Soc. London. 1893. p. 138. - Fig. 17.

107 , Pasuruan (Java), Kobus leg.

Mit einigem Zweifel betrachte ich das vorliegende Exemplar als das Männchen dieser von Austen auf 1 Weibchen von Buru gegründeten Art. Dasselbe ist ganz stahlblau, der Hinterleib aber mehr purpurfarbig. Auch die Stirne und das Untergesicht sind glänzend stahlblau, Fühler und Beine sind rothgelb, letztere nur am Ende der Hinterschienen etwas verdunkelt. Flügel mit schwarzbraunem Vorderrand und mit einem grossen, damit zusammenhängenden, mit der Spitze nach unten gerichteten dreieckigen Flecken von gleicher Farbe.

Körperlänge $12 \mathrm{~mm}$.; Länge der Flügel $9 \mathrm{~mm}$.

Das von Austen beschriebene Weibchen hatte eine schwarzgefärbte Hinterleibsbasis; die Zeichnung der Flügel und sonstige Merkmale stimmen wohl genügend mit meinem Männchen, um sie als beide Geschlechter derselben Art betrachten zu dürfen.

\section{CONOPIDAE.}

Conops javanicus Dol. Doleschall. Nat. Tijdschr. Ned. Indië. X. 409. Taf. IV. 'Fig. 2.

Sukabumi (Java), Fruhstorfer leg. $1 \sigma^{\top}$.

Der kurzen Beschreibung Doleschall's möohte ich noch Folgendes hinzufügen:

Fühler ganz gelblich-roth, von Thoraxlänge, das $1^{\text {te }}$ und $3^{\text {te }}$ Glied gleichlang, das 2 te anderhalb mal so lang als die übrigen. Untergesicht von derselben Farbe, mit gelblichweissem Schimmer. Stirne ganz bräunlich-roth, so wie auch der Hinterkopf. Rüssel an der Wurzel schwarz, grösstentheils jedoch bräunlich-gelb, $1^{1 / 2}$ mal so lang als der Kopf.

Thorax braunroth, mit 3 Längsstreifen, von welchen der mittlere sich ganz bis vorn erstreckt und daselbst etwas erweitert ist, hinten aber die Quernaht nur wenig überschreitet, die seitlichen sind vorn abgekürzt, erstrecken sich aber nach hinten bis nahe zum Schildchen. Schulterbeulen und Schildchen rothbraun, Hinterrücken braun, vorn mit Silberschimmer. Brustseiten namentlich oberhalb der Hüften etwas verdunkelt, mit nicht sehr starkem silberweissem Schimmer.

Auch an der Hinterleibsbasis findet sich ebensolcher Schimmer. Das 2te Segment zeigt vor dem Ende einen röthlichen Flecken; die folgenden Ringe sind beim vorliegenden Exemplare alle ziemlich verdunkelt, nur das Ende.des Abdomens ist wieder röthlich-braun.

An den Beinen sind die Schenkel und Schienen nur wenig verdickt. Hüften, namentlich die der Vorderbeine, sowie die Schienen an der Aussenseite mit Silberschimmer. Am Ende der letzteren findet sich eine abgeflachte Stelle, wie sie auch bei anderen Arten dieser Gattung, namentlich bei denjenigen, welche der Untergattung $\mathrm{Physocephala}$ angehören, vorhanden ist. Vorder- und Mitteltarsen schwarz, Hinterschienen sowie der übrige Theil der Füsse rothbraun. Krallen weiss, nur ganz an der Spitze schwarz.

Flügel gelblich-weiss, vor der Spitze etwas verdunkelt, Adern gelb.

Gewöhnliche Querader hinter der Mitte der Discoidalzelle, nur etwas jenseits der Mündung der Hilfsader. 
Macrochaeten fehlen fast ganz, es finden sich nur ein Paar kleinerer am Rande des Thorax. Körperlänge $14 \mathrm{~mm}$. ; Flügellänge $11 \mathrm{~mm}$.

Occem yia simillima n. sp. Tosari (Java), 1 ९, Kobus leg.

Stirn mattschwarz, mit glänzend schwarzem Stirndreieck, nur ganz vorn rothgelb, Untergesicht und Backen röthlichgelb, weissschimmernd. Fühler schwarzbraun, das 2te Glied so lang wie das

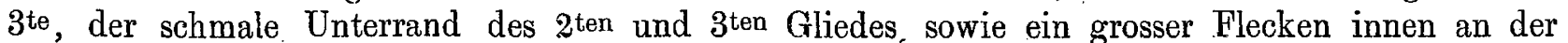
Wurzel des letzteren rothgelb; die Fühlerborste und der Rüssel schwarz.

Thorax schwarz, ohne deutliche Längsstriemen, Schulterbeulen und Brustseiten weisslich bestäubt, sowie auch die Hüften.

Hinterleib schwärzlich, mässig glänzend, die vorderen Ringe an den Seiten, der dritte Ring auch oben mit sehr schmalem, weissschimmerndem Hinterrande.

Schuppenförmiger Anhang des 5 ten Ringes gross, beiderseits braunroth, hinten mit schwarzem Rande, vorn glänzend.

Beine grösstentheils schwarzbraun, die Vorderhüften ausser der Wurzel, die Wurzelhälfte der Schenkel, ausser an der Oberseite, die Kniee röthlich-gelb. Auch die Hintertarsen an der Wurzel etwas lichter.

Flügel fast glashell, an der Wurzel kaum gelblich. Schwinger röthlich-gelb.

Körperlänge $5 \mathrm{~mm}$.; Flügellänge $4 \mathrm{~mm}$.

Die Art sieht der europäischen Occ. atrạ F. täuschend ähnlich; namentlich in der Grösse und in dem schwärzlichen Aussehen stimmt sie mit derselben sehr überein. Die weissen Binden am Hinterrande der Ringe sind aber, wenigstens beim vorliegenden Exemplare, schmäler, und die beiden letzten Fühlerglieder sind gleichlang, während bei Occ. atra das 2te Glied entschieden länger ist als das dritte. Auch ist der schuppenförmige Anhang des 5ten Hinterleibsringes grösser.

\section{MUSCIDAE.}

Ocyptera fuscipennis Wied. Aru (Rosenberg leg.) 1 Ex., Timor (Wienecke leg.) 2 Exx.; im Leydener Museum.

Das Exemplar von Aru stimmt in der Farbe des Hinterleibes mit der Wiedemann'schen Beschreibung überein. Von den auf Timor erbeuteten Exemplaren hat das eine auch am Hinterrande des $3^{\text {ten }}$ Ringes noch ein dreieckiges, schwarzes Fleckchen, während das zweite am 2 ten und $3^{\text {ten }}$ Ringe eine schwarze Mittellinie zeigt, und auch der Hinterrand dieser Ringe schmal schwarz ist.

Pse ud of ormosia moneta Gerst. Waigeu, 3 Exx., Bernstein leg. Im Leydener Museum.

Die Bestimmung der früher in die Gattung $R$ utilia gebrachten, durch die schönen Metalfarben sehr auffälligen Fliegen ist oft mit bedeutenden Schwierigkeiten verknüpft. Es ist hieran namentlich Walker Schuld, indem er auch besonders in dieser Gattung eine Menge von Arten beschrieb, ohne irgend welche Abbildung und meistens auch ohne die unterscheidenden Merkmale genügend hervorzuh eben. Weil die Beschreibungen überdies kurz gehalten sind, so bleibt man über die Identität seiner Arten meistens im. Unklaren; nur durch Untersuchung der Typen Walker's wäre hierüber Sicherheit zu erlangen.

Es liegen mir mehrere »Rutilien" vor; welche wohl zum 'Theil aller Wahrscheinlichkeit nach mit Walker'schen Arten identisch sind, welche aber aus obengenanntem Grunde augenblicklich des Fragezeichens nicht entbehren können. Ich lasse sie einstweilen bei Seite, möchte aber noch einige Bemerkungen machen über obige, von Gerstaecker ausführlich und gut beschriebene Art:

Es liegen mir nämlich 3 Exemplare vor, sämmtlich von Waigeu und in allen Merkmalen einander sehr ähnlich, aber durch die Farbe des Hinterleibes etwas unter einander verschieden. Eines der- 
selben stimmt ganz mit Gerstäcker's Beschreibung; den auch von diesem Autor wohl als zufällig betrachteten, unrégelmässigen, kupferrothen Flecken vermisse ich aber hier. Es zeigt dieses Exemplar also am $3^{\text {ten }}$ und 4ten Ringe je 2 , metallisch-grüne Flecken von etwas viereckiger Gestalt. Beim 2ten Exemplare erstrecken sich diese Flecken seitwärts viel weiter, sodass sie eine hinten stark eingebuchtete Binde bilden und mit der daselbst am Bauche befindlichen, metallgrünen Binde zusammenhängen. Dagegen ist letzteres beim dritten Exemplare am $4^{\text {ten }}$ Ringe, jedoch nicht am $3^{\text {ten }}$ der Fall. Die mittleren Längsstriemen des Thorax sind bei allen diesen Exemplaren sehr schmal und die Seitenstriemen vorn abgekürzt. Trotz der abweichenden Hinterleibsfärbung scheinen mir jedoch alle diese Exemplare einer und derselben Art anzugehören, weil sie sonst so sehr übereinstimmen. Daraus würde dann aber hervorgehen, dass auf die Hinterleibsfarbe auch wohl bei anderen Arten kein grosses Gewicht zu legen ist; so könnte z. B. R. lucigena Walk, mit einer Querbinde und 2 Paaren von Flecken am Abdomen wohl nur Varietät von 'R. callipygos Gerst. sein. Es wird aber von ersterer weisses Toment am Kopfe, von letzterer gelbes angegeben, sodașs die Identität doch immerhin zweifelhaft bleibt. Jedenfalls schien es mir erwünscht auf diese Thatsache hinzuweisen.

Der Kiel des Untergesichtes ist gerade bei Ps. moneta für diese Gattung schmal und erscheint dadurch auch nicht besonders platt und niedrig; wie Brauer es für dieselbe angiebt.

Haematobia exigua de Meij. de Meijere in: P. Schat. Verdere Mededeelingen over "Surra". Mededeel. Proefstation Oost-Java 3e Ser. No. 44. 1903. Pasuruan (Java), mehrere Exemplare auf Vieh gefangen (Schat leg.)

Eine kurze Beschreibung dieser Art wurde schon in obiger, sich auf die Surra-Krankheit beziehender Abhandlung aufgenommen. Seitdem habe ich von Herrn Schat ausgedehnteres Material bekommen und dadurch auch das Männchen kennen gelernt. Weil obige Abhandlung den auswärtigen Dipterologen schwer zugänglich sein wird, beschreibe ich hier die Art von Neuem in etwas ausführlicherer Weise.

Stirne des Männchens'sehr schmal mit' sehr schmalen weissen Periorbiten, zwischen welchen eine feine schwarze Linie sich befindet. Stirndreieck schwarz. Beim Weibchen wird die schwarze Stirnmittelstrieme durch gleichbreite weisse Periorbiten von den Augen getrennt.

Untergesicht weiss.

Fühler braungelb, namentlich das etwas verdickte 2te Glied dadurch auffallend, das 3te Glied am Ende verdunkelt. Alle Glieder mit weissem Schimmer.

Taster so lang wie der Rüssel, bräunlichgelb.

Rüssel braungelb, oben dunkel.

Thorax dunhelgrau, weisslich bestäubt; von vorn betrachtet mit 2 feinen schwarzen Längslinien; bei seitlicher Ansicht zeigen sich die Seiten des Rückens breit dunkel gesäumt. Brustseiten, Schulterbeulen, Quernaht und ein Querband vor dem Schildchen in bestimmter Richtung weisslich schimmernd.

Hinterleib dunkelgrau, mit schwarzer, am Ende des $3^{\text {ten }}$ Ringes schmal endender, und nur bei bestimmter Betrachtung ganz deutlich hervortretender Mittelstrieme. Bisweilen jederseits derselben noch Spuren von dunkleren Fleckchen.

Beine bräunlich-gelb, an der Aussenseite mehr oder weniger verdunkelt, bisweilen die Hinterbeine, mit Ausnahme der äussersten Wurzel von Schenkeln und Schienen, ganz dunkel, was bei den vorderen Beinen mit den Schenkeln bis nahe zur Spitze, dem mittleren Theile der Schienen und dem Ende der Tarsen der Fall sein kann.

Das Geäder wie bei H. irritans L.; die kleine Querader liegt noch gerade jenseits der Aussmündung der 1 ten Längsader. Schwinger und Flügelschüppchen weisslich.

Körperlänge $4 \mathrm{~mm}$; Flügellänge $4 \mathrm{~mm}$.

Nach den Untersuchungen des Herrn Schat spielt. die Fliege, zusammen mit Stomoxys calcitrans L., die Hauptrolle bei der Übertragung der Surra-Krankheit. 
Idia(?) melanostoma Wied. Ardjoeno (Java) 1 옹 Hekmeyer leg. Im Leydener Museum.

Der Kiel des Untergesichtes ist sehr wenig entwickelt, fast nur zwischen den Fühlerwurzeln als länglicher, ungefurchter Höcker vorhanden. Nach unten verliert er sich sehr bald; ist aber daselbst nicht schneidig. Die Art gehört vielleicht zu Idiella; um dies fest zu stellen wäre es aber nötig, das $\sigma^{7}$ zu untersuchen.

Sie sieht der ebenfalls einfarbigen Idiella unicolor ähnlich, ist aber mehr grünlich, letztere bläulich-grün; der Hinterleib ist relativ länger, stärker glänzend, bei S. unicolor weiss bereift; Backen und Brustseiten sind gelb-, bei I. unicolor weissbehaart. Schienen und Tarsen sind gelb; bei I. unicolor nur die hinteren Schienen gelblich.

Die Flügel zeigen einen deutlichen bräunlichen Anflug, namentlich sind sie an der Spitze und an der Basis des Vorderrandes gebräunt.

\section{Id iella limbipennis Macq. Pasuruan (Java), $1 \sigma^{7}, 2$ 우, Kobus leg.}

- Der Kiel des Untergesichtes ist wenig entwickelt, auch zwischen den Fühleren viel schmäler als bei anderen Arten dieser Gattung, sodass erstere kaum getrennt sind; unter denselben ist er eine kurze Strecke schneidig und verliert sich dann in das Untergesicht. Letzteres ist glänzend schwarz, oben weiss bestäubt. Klauen und Haftläppchen sind bei den Männchen etwas grösser als bei den Weibchen. Die Periorbiten sind weiss, die schwarzen Tupfen, welche die Borsten tragen, sind aber so gross, dass von der weissen Farbe fast nur noch eine netzartige Zeichnung übrig bleibt. Wangen glänzend schwarz; in der Mitte, zwischen Auge und Untergesicht, ein weisser Flecken. Die Augen des Männchens nur durch eine weisse Linie getrennt; Periorbiten daselbst mit einer Reihe von Borsten.

Beide Geschlechter zeigen am Hinterleib eine namentlich schräg von hinten betrachtet deutliche, dunkle Mittelstrieme. Sternopleuralborsten $1: 1$.

Idiella mandarina Wied. Ardjoeno (Java), 1 , , Heckmeyer leg.; Flores, 1 , , Semmelink leg.

Beim Exemplare von Flores erstreckt sich die gelbe Farbe des Hinterleibes auch über die Seiten des 3ten Ringes in der Gestalt dreieckiger, sich nach hinten verschmälernder Flecken. Das javanische Exemplar hat von denselben nur ganz vorn eine Spur; dagegen finden sich hier am 2 ten Ringe noch 2 Längsstriemen von der dunklen Farbe, welche sich sogar noch etwas auf den 1 ten Ring fortsetzen.

Idiella quadrimaculata Macq. Tosari (Java); $8 \sigma^{\top} \sigma^{\top}$ und 1 \&, Kobus leg.

Nach dem Kiele des Untergesichtes und den bei den $\sigma^{\top} \sigma^{\top}$ verlängerten Klauen und Haftläppchen zu urtheilen gehört auch diese Art wieder zu Idiella.

Der zwischen den Fühlern liegende Theil des ersteren zeigt in der Mitte eine Längsfurche.

Der obere und vordere Rand der Mesopleuren ist meistens etwas gelblich. Die Bauchseite des Abdomens ist gelb mit schwarzen Hinterrandsäumen und ebensolcher Spitze, letztere mit.weisser Bestäubung an den Seiten. Sternopleuralborstẹn $1: 1$.

Flügel an der Spitze, namentlich am Vorderrande, etwas verdunkelt. Bei dem von Macquart nicht gekannten W.eibchen ist diese Bräunung stärker ausgebildet als bei den Männchen. Die Flecken des Hinterleibes sind bei demselben wenig scharf begrenzt und also weniger deutlich. Die Periorbiten sind gelblich-grau bestäubt.

I diella unicolor Macq. Tosari (Java), 7 우, Kobus leg.

Der Kiel- setzt sich unten etwas weiter fort als bei I. melanostoma, verliert sich aber 
doch bald in das Untergesicht. Der die Fühler trennende Theil in der Mitte mit einer Längsfurche. Die Periorbiten finde ich gelblich-grau bestäubt; am unteren Theile der Wangen findet sich in der gelblich-grauen Bestäubung ein ovales, glänzend schwarzes Fleckchen. Oben an den Brustseiten eine breite, weisse Längsstrieme. Hinterleib mit weisslichem Schimmer und mehr oder weniger deutlicher dunkler Rückenstrieme und Einschnitten.

Sternopleuralborsten $1: 1$.

Idiella xanthogaster Wied. Ardjoeno (Java), $1 \sigma^{7}$, Hekmeyer leg. Im Leydener Museum.

Bei diesem Exemplare sind die Wurzel der Schienen und der Tarsen gelb, wie Osten Sacken es von einem Ambon'schen Exemplare angiebt. Nach Wiedemann sollen die Beine ganz schwarz sein. Der Kiel des Untergesichtes ist wenig entwickelt, nach unten nicht eigentlich schneidig.

Pyrellia chalybea Wied. Wiedemann. Aussereurop. zweiflügel. Insekten. II. 402. (Lucilia chalybea).

Von dieser Art liegt mir ein $q$ vor, welches mir ermöglicht, der Beschreibung Wiedemann's noch etwas hinzuzufügen. Zunächst ergiebt sich, das die Art der Gattung Py rellia angehört.

Hauptfarbe metallglänzend violet, je nach dem einfallenden Lichte ist die Farbe aber bald bronze, bald stahlblau oder metallisch roth.

Stirnstrieme schmal, vertieft, matt dunkelbraun; Periorbiten glänzend schwarz, nach hinten mehr violet. Wangen glänzend schwarz.

Zu beiden Seiten findet sich neben der Fühlerwurzel, nur wenig höher als diese, eine runde Makel mit weissem Schimmer und darunter, neben der Basis des 3ten Fühlergliedes, eine grössere ebensolche. Auch dieses Glied und die Fühlergrube zeigen weissen Schimmer.

Die Backen glänzend, mit rothem, grünem und violettem Metallglanz.

Beine glänzend schwarz.

Am Hinterleib finden sich keine Macrochaeten.

Hintere Querader stark S-förmig geschwungen, die 4te Längsader verläuft in einem Bogen zum Flügelrand. Die 3te Längsader zeigt nur an der Wurzel einige Härchen. Von Sternopleuralborsten findet sich nur eine, in der oberen Hinterecke. Hypopleuralborsten fehlen.

M usca modesta n. sp. Pasuruan (Java), mehrere Exemplare, meistens auf Vieh. P. Schat leg.

Schwarzbraun, mit gelblicher Bestäubung. Augen des Männchens fast zusammenstossend, die sehr. schmalen, weissen Periorbiten durch eine feine, schwarze Linie getrennt, welche sich nach vorn etwas erweitert. Untergesicht schwärzlich mit weisslichem Schimmer. Antennen schwarz, ebenfalls etwas weisslich schimmernd. Taster gelb. Thorax schwarzbraun mit gelblicher Bestäubung, mit 4 schmalen, glänzend schwarzen Längsstriemen; die inneren liegen weit auseinander und laufen ununterbrochen bis zum Schildchen durch; die äusseren sind ebenso breit wie die inneren, an der Quernaht abgebrochen, der vordere Theil liegt schief nach aussen gerichtet. Brustseiten wie die Oberseite des Thorax, sowie auch das Schildchen.

Abdomen kurz und breit, mit gelblicher Bestäubung, undurchsichtig; der Ite Ring bis auf einen schmalen Hintersaum schwarz, der 2te mit ebensolchem, in der Mitte mehr oder weniger dreieckig erweitertem Vorderrande, der 3te Ring mit dreieckigem, schwärzlichem Mittelflecke am Vorderrande. Flügel an der Wurzel etwas gelblich; die Adern gelb, nahe der Flügelspitze dunkler.

Schüppchen und Schwinger weisslich. Hintere Querader fast in der Mitte zwischen der kleinen Querader und der Beugung der 4ten Längsader; das Geäder sonst wie bei M. corvina F. Beine schwarz; die Mittelschienen an der Innenseite hinten mit einem kleinen Dorne hinter der Mitte. Krallen und Haftläppchen in beiden Geschlechtern klein, bei den $\sigma^{7}$ jedoch etwas grösser.

Das $q$ ist dichter bestäubt als das $\sigma^{\gamma}$, sodass bei demselben der Thorax auch senkrecht von oben betrachtet gelblichgrau erscheint, während er dann bei dem $\sigma^{\top}$ fast schwarzbraun aussieht. 
Die Mittelstrieme der Stirne ist mattschwarz und fast ebenso breit wie die Periorbiten. Besondere Orbitalborsten fehlen.

Körperlänge $6 \mathrm{~mm}$.; Flügellänge $5 \mathrm{~mm}$.

Die Art sieht der M. corvina F. ähnlich, ist aber durch die Farbe des Hinterleibes und durch die gelben Taster sogleich zu erkennen.

Die Borste an der Innenseite der Mittelschienen fehlt sonst bei Musca. Im Uebrigen passt die Art aber durchaus in diese Gattung, sodass ich sie bei der mangelhaften Kenntniss der tropischen Arten einstweilen in derselben belassen möchte.

Von den von älteren Autoren beschriebenen Arten scheint auch M. n ebulo F. der vorliegenden Art ähnlich zu sehen. Dieselbe hat jedoch ein gelblich durchsichtiges, in gewisser Richtung weissliches, fast gew ürfeltes Abdornen.

\section{CALOBATINAE.}

Nerius lineolatus Wied. Pasuruan (Java), mehrere Exemplare, Kobus leg.

Die Seitenstriemen des Thorax sind immer breiter als die mittleren Striemen und nur bei bestimmter, namentlich bei vorderer Ansicht deutlich von denselben abgetrennt.

\section{SEPSINAE.}

Sepsis javanica n. sp. Tosari (Java), mehrere Exemplare, Kobus leg. Fig. 18.

Glänzend schwarz, auch der Kopf. Die tiefe Fühlergrube des Untergesichtes schwärzlich, mit weisslichem Schimmer, die dazwischen befindliche Strieme mehr oder weniger gelblich, sowie auch der obere Mundrand und das Ende des Rüssels.

Fühler von matt-gelbbrauner Farbe, am Oberrand und aussen verdunkelt, mit weisslichem Schimmer.

Thoraxrücken weniger glänzend und etwas bräunlich bestäubt, vor der Flügelwurzel ein silberglänzendes Längsstreifchen; eben solche Flecken oberhalb der Hüften. Das Abdomen ganz glänzend' schwarz. Beine gelbbraun, nur das Ende der Schenkel und der Tarsen etwas verdunkelt. Beim Männchen die Vorderschenkel in der Mitte der Unterseite mit vorspringendem, stumpfem Höcker, die Schienen mit dementsprechender Einbuchtung.

Flügel hyalin ohne schwarzen Punkt, die 3te und 4te Längsader am Ende etwas, bisweilen aber sehr wenig, convergirend. Die Entfernung der beiden Queradern nur wenig grösser, als die Länge der hinteren Querader beträgt.

Körperlänge $4 \mathrm{~mm}$., Flügellänge $3 \mathrm{~mm}$.

\section{ORTALINAE.}

Xenaspis vespoides n. sp. Darjeeling (Bengalen), 5 Exx. Fig. 19, 20.

Einige der für diese Gattung von Osten Sacken angegebenen Merkmale treffen für die vorliegende Art nicht zu; weil diese aber sämtlich der einzigen bis jetzt bekannten Art X. polistes O. S. entnommen sind, so kann es nicht befremden, dass in der ausführlichen Gattungsdiagnose auch Speciescharaktere aufgenommen wurden. So ist bei meiner Art das Untergesicht auch vorn deutlich gerundet; die Fühlerborste ist nicht sehr kurz pubescent, sondern mässig lang gefiedert; der Thorax ist bedeutend länger als breit, fast zweimal so lang. Es finden sich 4 schwache Borsten vor dem Schildchen; letzteres tritt auch hier sehr wenig vor; es trägt ausser den 2 Endborsten noch jederseits 2 schwächere (bei einem Exemplar beobachtete ich deren an der einen 
Seite sogar 3). Das Abdomen ist an der Wurzel schmal; es erweitert sich nach hinten allmählich, ist also keulenförmig.

Kopf und Fühler rothgelb, mit einigem Glanze, namentlich die Wangen glänzend. Fühlergruben mit weisslichem Schimmer, am unteren Ende schwarz. Zwischen letzteren und den Augen findet sich jederseits ein gelbschimmernder Flecken. Das schmale, dritte Fühlerglied erreicht den Mundrand bei weitem nicht. Fühlerborste lang, jederseits mässig lang gefiedert; in der Endhälfte sind die Haare kurz.

Thorax gekörnelt, mattbraun, sehr kurz und dicht gelblich behaart, mit wenig hervortretendem, schmalem, schwarzem Mittelstreifen zwischen 2 dunklen Linien und 1 ebensolchem breiterem jederseits am Thoraxrande. Letztere begrenzen vorn die Innenseite der rothbraunen Schulterbeulen. Brustseiten rothbraun, mit einem grossen, dreieckigen gelben Flecken vor der Flügelwurzel und einem kleineren, ovalen neben dem Hinterrücken.

Schildchen klein, aber doch noch etwas mehr entwickelt als bei X. polistes, $1 \mathrm{~mm}$. lang, $3 \mathrm{~mm}$. breit, glänzend braungelb, nackt; Hinterrücken schwarzbraun, ebenfalls nackt und glänzend.

Hinterleib $1 \frac{1}{2}$ mal so lang als der Thorax, schwarzbraun, etwas glänzend, das 1 te Glied wenig. verlängert, grösstentheils rothbraun, das 2 te Glied nur an den Seiten von dieser Farbe; die Basis des Hinterleibes gelbbraun, die Endhälfte sehr kurz dunkel behaart. Auch beim $\&$ ist der 5te Ring. nicht versteckt. Legeröhre kurz, flach gedrückt.Beim ơ der keulenförmige Hinterleib hinten abgestutzt.

Beine rothbraun, die Schenkelwurzel und die Schienen auf der Mitte mehr oder weniger geschwärzt, namentlich die Hinterschenkel mit Ausnahme der Spitze schwarz.

Flügel grösstentheils rauchbraun, an der Spitze noch dunkler, der Vorderrand bis an die dritte Längsader braungelb, das Stigma nur wenig dunkler. Das Geäder stimmt ganz mit OstenSacken's Figur $5^{1}$ ), nur sind die $1^{\text {te }}$ und 2 te Längsader etwas länger und die hintere Querader ist weniger gebuchtet. Alle meine Exemplare zeigen die fremdartige. Bildung der sehr kleinen hinteren Basalzelle. Schwinger braungelb, der Knopf etwas verdunkelt.

Körperlänge $25 \mathrm{~mm}$.; Flügellänge $20 \mathrm{~mm}$.

Die Art ist durch die bedeutendere Grösse und durch die Farbe sogleich von X.polistes zu unterscheiden. Merkwürdigerweise zeigten die gesammten Exemplare eine Längsfaltung der

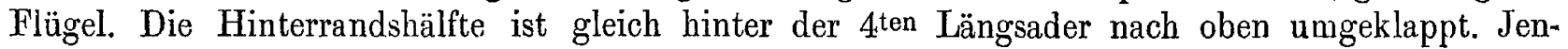
seits der $5^{\text {ten }}$ Längsader. biegt sich dann die Flügelfläche wieder mehr oder weniger allmählich nach unten um. Durch dieses an das Benehmen der Vespiden erinnernde Verhalten wird der Speciesnatme Osten Sacken's um so bezeichnender und auch der meinige deutet darauf hin. Auch bei Stenopterina lässt sich bisweilen eine ebensolche Faltung, aber weniger deutlich, beobachten. Mit dieser Gattung ist Xenaspis wohl nahe verwandt.

Bei den Vespiden wird der hintere Flügeltheil nach unten umgeklappt, die Analogie ist also eine unvollständige.

Zygaenula albofasciata n. sp. Aus Betelnusspalmen gezüchtet, Backergunge (Vorder Indien), einige Exemplare, Indian Museum, Calcutta. Fig. 21.

Kopf glänzend schwarz, zwischen Scheitel und Hinterkopf ein braungelber Querstreifen und auf der Stirne 2 längliche, braungelbe, dreieckige Fleckchen. Namentlich Stirne und Untergesicht stark glänzend. Fühler mit braungelben Wurzelgliedern, das dritte Glied lang und schmal, wenigstens oben und am Ende schwarzbraun. Borste ungefiedert.

Thorax schwarz,- mit einigem Glanze, zu beiden Seiten und in der Mitte mit einem, die Quernaht nicht überschreitenden, weissen Längsstreifen. Zu beiden Seiten des Mittelstreifens liegt ein spitzer, dreieckiger Flecken als Andeutung zweier weiterer Längsstriemen, gerade vor dem Schildchen.

Thoraxseiten glänzend schwarz mit grossem, länglichem, weissem Flecken. Dann findet sich noch ein kleiner ebensolcher gerade vor der. Flügelwurzel. Schildchen und Hinterrücken glänzend schwarz. Hinterleib an der Wurzelhälfte gelblich, die Endhälfte metallglänzend, violetschwarz, auf der Mitte scharf gekielt.

1) Osten Sacken. Diptera from the Philippine Islands. Berlin. Entomol. Zeitschr. XXVI, 1882, p. 220. 
Beine schwarz, die Kniee etwas bräunlich, die Tarsen gelblich-weiss.

Flügel glashell, die Wurzelhälfte dunkelbraun, mit lichteren Kernen in den Zellen.

Körperlänge $5 \mathrm{~mm}$; Flügellänge $6 \mathrm{~mm}$.

\section{TRYPETINAE.}

\section{Rhabdochaetan. g.}

Die Gattung steht der unlängst von Becker ${ }^{1}$ ) publicirten Gattung Schizopteron nahe, namentlich wegen des Kinschnittes am Flügelvorderrand. Doch finden sich mehrere Unterschiede, sodass es mir nicht möglich erschien, meine Art derselben einzuverleiben. Zunächst zeigt das Geäder manche Differenzen. Die 2te Längsader ist gerade, relativ lang. Die die Discoidalzelle distalwärts abschliessende Querader verläuft sehr schief nach unten und auch die Analzelle ist durch eine mehr schief liegende Ader begrenzt. Die $3^{\text {te }}$ und $4^{\text {te }}$ Längsader verlaufen fast parallel und die Flügelspitze ist gerundet.

Auch die Beborstung ist eine andere, und namentlich sind die Borsten sehr verschiedenartig, bald haarförmig, bald stark verdickt, die Farbe bald dunkelbraun, bald weiss. Solche Verschiedenheiten finden sich bei Schizopteron nicht, sonst hätte Becker dieselben wohl erwähnt.

Der Thorax zeigt 2 Dorsocentral- und 2 Praescutellarborsten, welche fast ebenso weit von einander entfernt sind als erstere; ferner jederseits 1 Humeral-, 1 Praesutural-, 2 Notopleural-, 1 Supraalar-, 1 Postalarborste, alle haarförmig und dunkel gefärbt; an den Pleuren finden sich 2 ebensolche nur etwas hellere und überhaupt schwache Mesopleural- und I Sternopleuralborste. Das Schildchen trägt 4 Borsten, von welchen die mittleren etwas kürzer, aber bis zur Spitze ziemlich dick und weiss gefärbt sind.

Der Kopf zeigt am Scheitel neben den Augen je eine lange, nach hinten gebogene, dunkle Borste und zwischen diesen beiden noch eine kurze, weissliche; vor den ersteren steht je eine kurze weissliche und ferner etwas mehr nach aussen 2 Fronto-orbitalborsten, von welchen die hintere sehr auffällig verdickt, oder besser abgeflacht ist und namentlich bei seitlicher Ansicht des Kopfes durch seine schwärzliche Farbe sehr hervortritt; das obere Ende ist etwas zugespitzt. Die vordere Frontoorbitalborste ist haarförmig, weiss, etwas nach innen gebogen. Der Ocellenfleck ist, wenn überhaupt vorhanden, doch äusserst unscheinbar. Vor demselben stehen noch 2 längere weisse, nach vorn gerichtete Borsten. Zwischen den Macrochaeten finden sich, wie am Thorax, zerstreute, aber ziemlich lange, weisse Härchen.

Unten an den Vorderschenkeln beobachtete ich etwa 5 längere Borsten, sowie aussen an denselben und an den Hinterschienen einige kürzere; sonst sind die Beine fast nackt.

Wie bei Schizopteron sind Stirne und Mundrand vorgezogen; das Untergesicht ist kurz und sehr stark concav.

Die Taster sind sehr auffallend breitgedrückt; die Augen gross, etwas höher als breit, die Backen entschieden schmal; der Rüssel dick, die Saugflächen schmal, aber kaum verlängert. Durch das Geäder und die eigenthümlichen Borsten zeigt die Gattung Annäherung an Ceratitis.

Rhabdochaeta pulchella.n. sp. Pasuruan (Java), aus Blüthenköpfchen von Blumea lacera D. C. gezüchtet, Kobus leg. Fig. 22, 23.

Kopf gelblich-weiss; die Stirne etwas mehr graulich, mit mehreren weissen Borsten, und überdies 2 schwarzen jederseits neben den Augen; namentlich die vordern sind relativ stark, die hintern stehen ganz nahe dem Hinterkopf.

Fühler gelblich-weiss, das 3te Glied lang und schmal, aussen concav, am Ende ziemlich spitz.

Taster bandförmig, weiss mit feinem, schwarzem Rande.

Thorax oben aschgrau bis dunkelbraun, vor dem Schildchen etwas lichter, mehr gelblich; zwischen beiden Farben gibt es jedoch keine scharfen Grenzen. Schildchen gelblich, an den Seiten etwas dunkler, mit 4 Borsten, die ausseren lang, mit dunkler Spitze, die inneren kürzer, weiss, relativ

1) Becker, $\Lambda$ egyptische Dipteren. Mittheilungen aus dem Zoolog. Museum zu Berlin, Il. 1903 p. 137. 
stark. Schulterbeulen gelblich, Thoraxseiten von derselben Farbe, mit vier runden, grauen Flecken hinter einander, von welchen der erste unter den Schulterbeulen liegt. Hinterrücken dunkelgrau. Jederseits 2 Dorsocentralborsten.

Hinterleib gelb, mit etwas röthlichem Anfluge, und mehr oder weniger deutlichen, in der Mitte unterbrochenen dunklen Querbinden, je eine an jedem Ringe.

Legeröhre ziemlich kurz und breit, glänzend bräunlich-gelb, am Ende mit einem schmalen, schwarzen Rande.

Beine gelblichweiss, die Mittel- und Hinterschenkel mit 2 schmalen, schwarzen Ringen, bisweilen auch an den Vorderschenkeln eine Spur von denselben. Auch an den Hinterschienen findet sich bisweilen ein wenig hervortretender, dunklerer Ring. Die Vorderschenkel tragen unten mehrere lange Borsten, sonst finden sich solche an den Beinen nicht.

Flügel mit verwickelter zierlicher Zeichnung, der mittlere Theil gelbbraun, mit 3 helleren, meistens sogar ganz glashellen grossen Flecken; neben dem oberen liegen ein schwarzer und weiter viele kleinere glashelle Fleckchen. Im breiten glashellen Flügelsaume verlaufen radienartig von der mittleren Partie ausgehende, sich allmählich verjüngende Streifen, von welchen ein Theil den Flügelrand erreicht. Am Flügelvorderrande wechseln solche mit kürzeren regelmässig ab; die, welche den Rand erreichen, sind dunkel gesäumt, die übrigen weiss-gesäumt.

Der dem Hinterrand zunächst liegende Theil des Flügels erhält durch mehrere helle Flecken ein wolkiges Aussehen.

Die $1^{\text {te }}$ Längsader zeigt mehrere Borsten, an der $3^{\text {ten }}$ beobachtete ich deren nur eine. Körperund Flügellänge $2,5 \mathrm{~mm}$.

Merkwürdigerweise wird die Färbung der Flügel hier nicht durch diffuse Färbung des Chitins hervorgerufen, sondern durch nur an den gefärbten Theilen vorhandene äusserst kurze, stabförmigen Härchen. An den weissen Stellen sind diese glashell, an den dunkleren sind sie mehr oder weniger verdunkelt. Bei mehreren anderen Trypetinen, welche ich daraufhin untersuchte (Carphotricha pupillata Fall., Tephritis pantherina Fall. Urellia stellata Füssl. Acidia heraclei L., Oxyphorá miliaria Schr. fand ich dagegen immer nur diffuse Färbung der Flügelhaut selber. Dagegen fand ich diese eigenthümlichen Härchen in ganz derselben Weise bei Schizopteron Möbiusi Beck., von welcher Art mir Herr Becker in liebenswürdigster Weise ein Exemplar zur Untersuchung überliess, wofür ich ihm auch an dieser Stelle bestens Dank sage. Es ergab sich, dass hier der farbige 'Theil der Flügel eine diffuse braune Chitinfärbung aufweist, dass aber überdies an allen schwarzgefärbten Stellen dunkle, stabförmige Härchen vorhanden sind. Bei den lackrothen Flecken in der Flügelmitte ist nur die Chitinschicht diffus braun gefärbt; bei durchfallendem Licht sind diese röthlich, bei auffallendem Licht sehr dunkelbraun. Der obere runde Flecken, welcher gerade unter dem Ende der 2ten Längsader liegt, ist überdies beiderseits mit glashellen stabförmigen Härchen besetzt. Infolgedessen erscheint dieser Flecken bei durchfallendem Lichte graulich-weiss, bei auffallendem Lichte braungelb. Die Härchen finden sich namentlich an der Oberseite; unten sind sie nur stellenweise vorhanden, die der Oberseite schimmern hier jedoch überall durch.

Rioxa Dúnlopi v. d. Wulp. - Van der Wulp. Tijdschr. v. Entomol. XXIII. p. 186. (Ptilona Dunlopi). - Java, 2 Exx. (Piepers); Ardjoeno (Java), 1 Ex. (Hekmeyer).

Die vorliegenden Exemplare setzen mich in den Stand, der auf 19 gegründeten Beschreibung van der Wulp's Folgendes hinzuzufügen: Der weisse Flecken in der oberen Wurzelzelle bildet kein constantes Merkmal, derselbe fehlt bei 2 der obigen Exemplare. Die 2te und 3te Längsader verlaufen bisweilen etwas wellenförmig. Die Analzelle ist hinten nicht durch eine schiefliegende, gerade Querader begrenzt, wie es aus van der Wulp's Figur hervorgehen würde, sondern dieselbe ist unten zipfelartig vorgezogen, wie auch bei Ptilona brevicornis v.d. Wulp; dies ist auch bei der Type van der Wulp's der Fall. Bei letzterer ist die braune Ausdehnung der Farbe in der 3ten Hinterrandzelle auch nicht ganz richtig angegeben; der grosse weisse Flecken in derselben liegt viel weniger schief.

Auch die Grösse bildet gegenüber Pt. brevicornis kein sicheres Merkmal. Während bei letzterer die Flügel $9.5 \mathrm{~mm}$. Länge erreichen können, sind sie bei der Type von Pt.Dunlopi 
nur $5 \mathrm{~mm}$. lang, bei einem der obigen Exemplare jedoch schon $8,5 \mathrm{~mm}$. - Auch ist bei meinen Exemplaren die Fühlerborste fast nicht kürzer gefiedert als bei Pt. brevicornis.

Constante Merkmale sind jedoch: das Fehlen des Randdorns, die Lage der hinteren Querader, indem bei R. Dunlopi von den distalen Ecken der Discoidalzelle die obere eine gerade, die untere eine schiefe ist, während sich bei Pt. brevicornis die Sache gerade umgekehrt verhält, ferner auch die von van der Wulp in der Tabelle auf p. 184 der citirten Arbeit angegeben Unterschiede in der Kopfform und die relative Länge des 3 ten Fühlergliedes.

Am Schildchen von R. Dunlopi beobachtete ich noch jederseits zwischen den 2 langen Borsten eine etwas feinere," oft von bedeutender Länge, bei Pt. brevicornis scheint diese ganz zu fehlen. Dadurch schliesst sich die vorliegende Art der Diagnose von Rioxa an. Dass wir es hier mit einer Rioxa zu thun haben, dafür spricht auch Folgendes: es ist eine Praesuturalborste vorhanden, die dritte Längsader ist an der Basalhälfte nicht nackt, sondern sehr kurz beborstet. Mit Ptilona hat sie aber die beiderseits gefiederte Fühlerborste gemein; auch liegt das Ende der 1 ten Längsader fast in gleicher Entfernung von den Enden der Hilfs- und der 2 ten Längsader.

Von den von Osten Sacken ${ }^{1}$ ) angeführten Unterscheidungsmerkmalen stimmen also 2, 3, 4 und 5 mit Rioxa, und nur die beiderseits gefiederte Fühlerborste spricht für Ptilona. Wenn man, namentlich wegen der Beborstung, beide Gattungen beibehalten will, so ist die Art also am besten bei Riox a unterzubringen.

Es hat schon Osten-Sacken darauf hingewiesen, dass nach van der Wulp's Beschreibung "P til o n a" sexmaculata v. d. W. in den Merkmalen 1 und 4 mit Rioxa übereinstimmt, und also auch wohl besser in letztere Gattung eingereiht wird. Einige der van der. Wulp'schen Exemplare liegen mir vor und zeigen mir, dass diese Art auch in den Merkmalen 2, 3 und 5 mit Rioxa stimmt, also ganz und gar dazu gehört.

Ptilona notabilis v. d. W. dagegen stimmt in Allem mit Ptilona, nur sehe ich feine Börstchen an der Wurzelhälfte der 3 ten Längsader; auch dieses bietet also keinen zuverlässigen Unterschied zwischen beiden Gattungen, um so weniger, als sie bei dem mir vorliegenden Exem-" plare von $\mathrm{Pt}$. brevicornis ebenfalls vorhanden sind, wenn auch äusserst klein.

Die ebenfalls eine beiderseits gefiederte Fühlerborste besitzende "Ptilona" modesta F. gehört nach den sonstigen Merkmalen ${ }^{2}$ ) auch wohl eher zu Rioxa; van der Wulp ${ }^{3}$ ) stellt sie zu Ptilona, besonders wegen der kurzen Subcostalader und des weniger gewölbten Thoraxrückens, durch welche Merkmale er die beiden Gattungen unterschieden haben will. Doch scheinen mir diese von geringfügigerer Natur als die Beborstungverhältnisse (das Verhalten der Präsuturalborste und der Schildchenborsten), welche ich einstweilen als trennende Merkmale beibehalten möchte. Nach diesen Merkmalen sollten von den von Kertész ${ }^{4}$ ) fraglich zu Ptilona gestellten Arten variabilis Kert. und Bischofi Kert. dieser Gattung auch wirklich angehören, Iateralis Kert. und quadrifera Walk. aber zu Rioxa zu stellen sein.

Im Übrigen scheint mir die Beborstung des Kopfes u. s. w. wohl für die Arten verschieden, aber nicht geeignet, um dieselben generisch zu trennen. Ocellarborsten fehlen bei Pt. brevicornis und P.t. sexmaculata, sie sind klein bei R. Dunlopi. Pt. brevicorn is hat 2 Mesopleural-, 1 Sternopleural-, 0 Pteropleuralborsten; R. Dunlopi 2 Mesopleural-, 1 Pteropleuralborste; Pt. Bisch ofi hat aber auch wieder die Pteropleuralborste.

\section{SAPROMYZINAE.}

\section{Sapromyza obscura. n. sp. Tosari (Java), 2 Exx., Kobus leg.}

Schwarz; Stirne matt schwarzbraun, die Periorbiten und das Scheiteldreieck grau; letzteres erstreckt sich fast bis an die Fühlerwurzel.

1) Osten Sacken: Diptera from the Philippine Islands. Berlin. Entomol. Zeitschr. XXVI. 1882. p. 226.

2) Osten Sacken, l.c. p. 227.

3) Van der Wulp. Tijdschr. v. Entomol. XLI. p. 219.

4) Kertész. Neue und bekannte Dipteren in der Sammlung des ungarischen National-Museums. Termész. Füz. XXIV. 1901 , p. $426-431$. 
Fühler dunkelbraun, mit gelblichem Anfluge, das $3^{\text {te }}$ Glied $1 \frac{1}{2}$ mal so lang wie breit; oval, am Ende abgerundet, die Borste mässig lang gefiedert. Untergesicht und Rüssel grau.

Thorax grau, bei bestimmter Ansicht mit Spuren von 4 sehr breiten dunkelbraunen Längsstriemen. Zahlreiche Reihen von Acrostichalbörstchen vorhanden.

Die Pleuren und der Hinterleib schwarzbraun, etwas glänzend.

Beine schwarzbraun, die Schienen vorn an der Wurzel etwas lichter, die Tarsen gelbbraun.

Flügel glashell, die Adern lichtbraun.

Schwinger gelblichweiss.

Körperlänge 4,5 mm.; Flüggellänge $5 \mathrm{~mm}$.

Wegen der schwarzen Farbe wäre die Art am ehesten mit S. signatifrons Kert. aus NeuGuinea zu verwechseln. Letztere zeigt aber ein gelbes Querbändchen am vorderen Stirnrande, und die Schwinger sind schwarzbraun mit gelbem Stiele.

Die ebenfalls ähnliche Homoneura picea v. d. Wulp lässt sich durch die gelbgefärbten Flügel, die einander sehr genäherte Hilfs- und Subcostalader, den schwarzen Endknopf der Schwinger und die länger gefiederte Fühlerborste unterscheiden.

\section{CHLOROPINAE.}

Pseudeurina n. g.

Stirne vorgezogen, flach, dicht kurz behaart, die sehr kurzen Fühler darunter versteckt, das 3te Glied derselben rundlich, am Ende spitz; die Borste pubescent.

Untergesicht stark zurückweichend, die beiden ovalen Fühlergruben nur durch einen schmalen Kiel : getrennt. Taster cylindrisch. Saugflächen des Rüssels kurz. Augen etwas länger als breit, kurz behaart.

Schildchen am Rande beborstet, namentlich daselbst in der Mitte mit 2 längeren Borsten.

Hinterleib, so wie der Thorax nur sehr kurz behaart, der lte Ring nicht besonders verlängert.

Hinterschenkel nicht verdickt; Hinterschienen gekrümmt, am Ende ziemlich dick, ohne Dornen.

Randader bis zur 4ten Längsader fortgesetzt; letztere biegt sich vom Ende der Discoidalzelle an etwas nach oben und verläuft der 3 ten Längsader parallel.

Durch die Kopfbildung steht die Gattung der Gattung Eurina nahe. Sie unterscheidet sich von derselben durch das zugespitzte $3^{\text {te }}$ Fühlerglied, durch die bis zur $4^{\text {ten }}$ Längsader fortgesetzte Randader und durch die Borsten des Schildchens.

Pseudeurina maculata n. sp. Pasuruan (Java), 4 Exx., Kobus leg. Fig. 24, 25.

Kopf blassgelb, Stirne braun, mit schmalen weisslichen Augenrändern und ebenso gefärbtem Scheiteldreieck, welches sich fast bis an die Fühler in feiner Linie fortsetzt, durchaus matt und dicht, aber sehr kurz behaart. Nur der Ocellenhöcker schwarz. Fühler braungelb, das dritte Glied am oberen Rande etwas verdunkelt.

Thorax blassgelb, matt, mit 3 breiten, nur durch feine, gelbe Linien getrennten, matten, dunkelbraunen Längsstriemen. Schildchen bräunlich, am Rande gelb. Hinterrücken glänzend schwarz. Brustseiten etwas glänzend, gelb mit etwa 3 schwarzen Fleckchen.

Hinterleib mässig glänzend, blassgelb mit schwarzen Hinterrändern an den Segmenten und ziemlich breiter, schwarzer Mittelstrieme, welche sich in jedem Segmente hinten erweitert. Es bleibt somit von der Grundfarbe in jedem Segmente jederseits ein halbkreisförmiger Flecken übrig, indem auch die schwarzen Hinterränder sich nach den Seiten hin stark erweitern. Der Bauch ganz gelb. Die vorderen Beine ganz blassgelb, nur die letzten Tarsenglieder verdunkelt. Hinterbeine mit schwarzem Wische am Ende der Schenkel, die Endhälfte der etwas gebogenen und namentlich am Ende ziemlich dicken Schienen ganz schwarz, ebenso wie die 3 letzten Tarsenglieder.

Flügel glashell; die Entfernung der beiden Queradern etwa doppelt so gross wie die Länge der hinteren Querader.

Körperlänge $4,5 \mathrm{~mm}$. Flügellänge $4 \mathrm{~mm}$. 
Myrmemorpha rufescens n. sp. Pasuruan (Java), einige wenige Exemplare; Kobus leg. Fig. 26.

Im Habitus der M. cornuta Fall. sehr ähnlich.

Kopf weissgelb, mit grossem, fast die Fühlerwurzel erreichendem, glänzend schwarzem Scheiteldreieck. Fühler gelbbraun, der Oberrand verdunkelt, das dritte Glied oval, mit dicker, schwarzer Borste von doppelter Fühlerlänge. Thorax rothbraun, mit dem Anfang einer schwarzen Mittelstrieme; dieselbe setzt sich höchstens bis auf die Mitte des Thorax fort, ist aber bisweilen kaum angedeutet. Zu beiden Seiten derselben eine feine, weisse Längslinie. Schulterbeulen mit einem schwarzen Fleckchen und einem ebensolchen etwas mehr nach hinten: auch diese Fleckchen sind jedoch nicht bei allen Exemplaren erkennbar. Brustseiten gelbbraun.

Hinterleib oben glänzend schwarz, an der Wurzel bisweilen heller gefärbt.

Beine braungelb, höchstens die Tarsen verdunkelt.

Flügel ungefärbt, die Queradern sind einander stark genähert.

Körperlänge $3 \mathrm{~mm}$; Flügellänge $2 \mathrm{~mm}$.

Hippelates bilineatus n. sp. Pasuruan (Java), aus Blüthenköpfchen von Blumea lacera D. C. gezogen, Kobus leg. Fig. 27, 28.

Kopf gelb, das Scheiteldreieck glänzend mit schwarzem Ocellenpunkt. Neben den Augen eine Reihe gelber Härchen. Augen sehr gross, höher als breit; Backen sehr schmal, mit einem Paar blassgelber Borsten. Taster und Fühler gelb, das runde dritte Glied der letzteren ganz am Ende, namentlich an der Aussenseite schwärzlich. Fühlerborste dunkel, pubescent. Rüssel mit etwas verlängerten schmalen Saugflächen. Untergesicht sehr kurz; die Knebelborsten blassgelb.

Thorax ockergelb, glänzend, nebst dem Hinterleib kurz gelblich behaart, mit 4 fast gleichbreiten Längsstriemen, von welchen jedoch die beiden inneren nur wenig dunkler sind als die Grundfarbe und nur ganz vorne, oberhalb des Halses, schwarz; letzteres ist aber nicht einmal bei allen Exemplaren der Fall. Die seitlichen, also weit aus einander liegenden, Striemen sind schwarz, an der Quernaht schmal unterbrochen und vor derselben um weniges breiter als dahinter. Der vor der Quernaht liegende Theil bildet ein fast ovales Fleckchen.

Thoraxrücken fast ohne Borsten; es finden sich nur am Rande einige Borsten, sowie auch ein Paar weit aus einander stehende vor dem Schildchen. Letzteres, sowie auch die Brustseiten gelb. Von den 4 Borsten des Schildchen sind die mittleren bedeutend länger als die seitlichen. Hinterrücken dunkelbraun bis schwarz. Schwinger gelblich-weiss.

Abdomen oben verdunkelt, grösstentheils glänzend dunkelbraun. Bauch gelb.

Flügel farblos, der Aderverlauf zeigt das gewöhnliche Verhalten, die 3te und 4te Längsader parallel, die $5^{\text {te }}$ erreicht den Hinterrand nicht. Die Entfernung der beiden Queradern etwas grösser als der halbe Abstand zwischen der hinteren Querader und dem Flugelrand. Beine ganz gelb, am Ende der Mittel- und Hinterschienen findet sich an der Innenseite eine schwarze Borste, von welchen namentlich die der Hinterschienen stark entwickelt ist.

Körperlänge 2,5 bis $3 \mathrm{~mm}$; Flügellänge $2 \mathrm{~mm}$.

Hippelates nigricornis Thoms. erscheint nahe verwandt, kann aber nicht dieselbe Art sein, indem das 3te Fühlerglied dunkelbraun, von gleicher Farbe wie die Borste sein soll. Diese Art hat ebenfalls 2 braune Striemen am Thoraxrücken; ob dieselben ebenfalls weit auseinander stehen geht aus der Beschreibung nicht hervor.

\section{GEOMYZINAE.}

Scyphella pumilio n. sp. Pasuruan (Java), 5 Exx., aus mit dem Gespinnst einer Motte überzogenen Blüthenköpfen von Blumea lacera D. C. gezüchtet, Kobus leg. Fig. 29. 
Die sehr kleine Art steht den europäischen Arten Sc. flava L. und flavella Zett. welche ebenfalls ganz gelbe Fïhler besitzen, sehr nahe. Von ersterer ist sie verschieden durch geringere Grösse, von beiden durch das oberseits verdunkelte Abdomen, durch die noch stärker genäherten Queradern und durch die weiter vom Flügelrande entfernte hintere Querader. Stirn blassgelb mit schwarzem Ocellenpunkt; Untergesicht weiss, am unteren Rande weniger eingebuchtet als bei Sc. flava. $3^{\text {tes }}$ Fühlerglied rund, ganz gelb; Fühlerborste schwarz. Stirne bis vornhin beborstet; Mundrand mit ziemlich langen Härchen, aber ohne eigentliche Knebelborsten.

Thoraxrücken mehr rothgelb, mit mässigem Glanze. Brustseiten und Beine blassgelb. Schildchen mit 4 Borsten.

Die Oberseite des Abdomens mehr oder weniger verdunkelt, bald glänzend rothbraun, bald schwärzlich. Der Vorderrand und der vordere Theil der Seitenränder an den Hinterleibssegmenten . gelblich.

Flügel glashell, mit gelben Adern; die Queradern sind einander sehr stark genähert. Schwinger meistens mit schwefelgelbem Knopfe.

Körperlänge 1,5 mm.; Flügellänge 1,25 mm.

Drosomyia n. g.

In der Körperform mit Drosophila übereinstimmend. Kopf niedrig und breit; ausser den 2 Paaren von Vertikal- und den 2 Ocellarborsten noch jederseits 3 Fronto-orbitalborsten. Fühler kurz, das 3te Glied oval, das 2te oben und unten mit J, resp. 2 langen Borsten. Fühlerborste. lang, beiderseits mässig lang gefiedert, mit zahlreichen Strahlen. Knebelborsten fehlen. Taster cylindrisch.

Thorax vorn gewölbt, jederseits 3 Dorsocentralborsten, 1’Humeral-, 1 Praesutural-, 2 Supraalar-, 1 Notopleural-, I Postalarborste. Schildchen mit 4 Borsten. Brustseiten mit I Prothorakal-, 1 Mesopleural- und 2 Sternopleuralborsten, von welchen eine (die vordere) jedoch schwach ist.

Hinterleib oval, noch etwas kürzer als der Thorax, namentlich am Ende mit ziemlich langen Borsten. Beine alle mit deutlicher Praeapicalborste, Vorderschenkel an der Aussen- und Unterseite mit einer Reihe langer Borsten; Mittelschenkel mit einer Reihe kürzerer Borsten; Hinterschenkel vor dem Ende mit einer nach aussen gerichteten Borste. Mittelschienen mit 2 Endspornen, einem langen und einem kürzeren. Flügel-relativ breit. Hintere Basalzelle ganz rudimentär, undeutlich abgegrenzt. Analzelle äusserst klein. Kleine Querader weit vor der Flügelmitte:

Von Drosophila, mit welcher sie den Habitus gemein hat, ist die Gattung sogleich durch

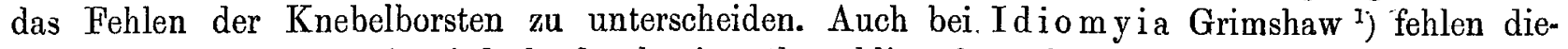
selben; diese Gattung ist jedoch durch eine überzählige Querader oberhalb der hinteren ausgezeichnet, auch zeigt die Fühlerborste weniger, aber längere Kammstrahlen und ist dieselbe unten an dem Basaltheile eine Strecke lang ganz nackt.

Drosomyia picta n. sp. Tosari (Java), mehrere Exemplare, Kobus leg. Fig. 30, 31.

Stirne bräunlichweiss mit schwarzem Ocellenpunkte, davor 2 parallele, braune Längsstrichelchen, welche die vordere Stirngrenze nicht erreichen.

Untergesicht weisslich, mit einem aus 3 neben einander liegenden, zusammengeflossenen Fleckchen gebildeten, braunen Querbändchen über dem Mundrand.

Fühler bräunlichweiss, das 3te_Glied oval, die grössere Endhälfte dunkelbraun.

Thorax bräunlichweiss, in der Mitte vor der Quernaht mit 2 braunen, dreieckigen, mit der Spitze nach vorn gerichteten, braunen Fleckchen; hinter derselben sind die Macrochaeten auf schwarzen Punkten eingepflanzt. An beiden Seiten des Thorax noch 2 ebensolche braune Fleckchen, welche etwas mehr nach vorn liegen als die mittleren Flecken, und ferner 2 ebensolche jederseits zwischen den inneren und äusseren Borstenreihen.

Brustseiten weisslich, dicht dunkelbraun gefleckt, sodass von der Grundfarbe nur wenig übrig bleibt.'

1) Grimshaw, Fauna Hawaiiensis III. 1: Diptera. p. 50. 
Hinterleib schwarzbraun, weissgefleckt; der lte Ring grösstentheils weiss, mit schwarzen Fleckchen auf der Mitte und schmalem, schwarzem Hinterrande; die folgenden Ringe schwarzbraun mit 4 halbkreisförmigen Flecken am Vorderrande, von welchen die mittleren bei weitem die grössten sind. Der letzte Ring an der Oberseite ganz weiss.

Bauch dunkelbraun mit hellen Einschnitten. Beine grösstentheils blassgelb, die Mittel- und Hinterschenkel mit Ausnahme der Spitze schwarzbraun.

Flügel grösstentheils dunkel mit zahlreichen glashellen Fleckchen; von welchen die grössten, mehr dreieckigen am Flügelrande liegen; 2 derselben finden sich nahe vor der Mündung der Iten Längsader, 4 liegen $z$ wischen der $1^{\text {ten }}$ und 2 ten Längsader, das $1^{\text {te }}$ weiter von den übrigen entfernt, letztere in fast gleichen Abständen. Ferner finden sich zwischen der 2 ten und 3 ten Längsader 2, zwischen der $3^{\text {ten }}$ und $4^{\text {ten }} 3$ ebensolche Fleckchen; am Flügelhinterrand folgen dann deren noch 5.

Zwischen den Längsadern finden sich meistens je 2 Reihen kleinerer Tropfenfleckchen, nur dem Flügelrande näher werden die Reihen zahlreicher, je 3-4. Die Queradern sind von einem ganz dunklen Hof umgeben. Das Flügelpigment besteht hier aus äusserst feinen und zahlreichen Körnchen in der Chitinschicht. - Schwinger! weiss.

Körperlänge $4 \mathrm{~mm}$., Flügellänge $4 \mathrm{~mm}$.

Drosophila nigropunctata v. d. Wulp. - Van der Wulp. Eenige uitlandsche Diptera, Tijdschr. v. Entomol. XXXIV p. 216. - Pasuruan (Java), aus dem mit Gespinnst einer Motte umgebenen Blüthenköpfchen von Blumea lacera D C. gezüchtet. 2 Exx. Kobus leg.

Die beiden Exemplaren sind heller als dạs von van der Wulp beschriebene, die Grundfarbe mehr weisslich mit braunen Fleckchen; wahrscheinlich sind sie noch nicht ganz ausgefärbt. Die Praeapicalborste ist auch an Vorder- und Hinterschienen vorhanden, aber äusserst zart und an den Vorderbeinen kurz. 


\section{ALPHABETISCHES VERZEICHNIS.}

\begin{tabular}{|c|c|c|c|c|c|c|c|c|}
\hline Argyramoeba distigma Wied. & & & $\begin{array}{l}\text { Seite. } \\
97\end{array}$ & Negritomyia spec. & & & & $\begin{array}{r}\text { Seite. } \\
96\end{array}$ \\
\hline xona chalcopygus Wied. & & & 100 & Nerius lineolatus Wied. & & & & 107 \\
\hline Baccha pulchrifrons Aust. & & & 102 & Occemyia simillima n. sp. & : & & & 103 \\
\hline " refulgens Aust. & & & 102 & Ocyptera fuscipennis Wied. . & & & & 103 \\
\hline Bibio obscuripennis n. sp. & • & & $8^{\prime} 6$ & Pachyrrhina dorsalis n. sp. . & & & & 89 \\
\hline eria trinotata n. sp. . & & - & 97 & fallax n. sp. . & - & & & 90 \\
\hline hrysopila simplex n. sp. . & & . & 97 & dimidiata n. sp. & • & & & 90 \\
\hline onops javanicus Dol. .. & - & & 102 & scurroides n. sp. & & & & 90 \\
\hline Conosia irrorata Wied. . & . & & 92 & Paragus serratus F. & & & & 99 \\
\hline Dicranomyia Kobusi n. sp. & . & & 91 & Plecia fulvicollis $\mathrm{F}$. & & & & 86 \\
\hline Drosomyia picta $\mathrm{n}$. sp. & $x$ & - & - $\quad 114$ & lugubris n. sp. & - & & & 86 \\
\hline ctata v. d. & . W & & $\ldots 115$ & melanaspis Wied. . & & & & 86 \\
\hline Eriocera nig & . & - & 92 & Pselliophora insignis $\mathrm{n}$. sp. & & & & 87 \\
\hline Eristalis argyrocephalus Macq. & . & & 100 & nigrithorax n. sp. & & & & 87 \\
\hline nitidus v. d. W. & - & & 100 & stigmatica n. sp. & & & & 88 \\
\hline splendens Le Guill. . & $\therefore$ & - & 101 & Pseudeurina maculata n. sp.. & . & & & 112 \\
\hline admeta bru & . & - & 94 & Pseudoformosia moneta Gerst. & . & & & 103 \\
\hline umerus aurifrons Wied. & $\cdot$ & & 99 & Ptecticus melanurus Walk. . & & & & $9 \check{9}$ \\
\hline Haematobia exigua de Meij.. & . & & 104 & » tricolor $\mathrm{n} . \mathrm{sp} . \quad$. & & & & 95 \\
\hline Helophilus curvigaster Macq. & - & & 99 & Ptilocera quadridentata. & & & & 96 \\
\hline Hermetia fenestrata $n$. sp. & . & & 93 & Pyrellia chalybea Wied. & & & & 106 \\
\hline laeta n. sp.. . . & - & - & 93 & Rhabdochaeta pulchella n. sp. & & & & 109 \\
\hline Hippelates bilineatus n. sp. & • & & . 113 & $\mathrm{Rl}$ & & & & 101 \\
\hline dia (?) melanostoma Wied. & & & 105 & ra n. sp. & & & & 92 \\
\hline liella limbipennis Macq. & & & 105 & Rioxa Dunlopi v. d. Wulp. . & & & & 110 \\
\hline mandarina Wied. . & - & - & 105 & Rosapha bimaculata n. sp. & & & & 96 \\
\hline quadrimaculata Macq. & $\theta^{2}$ & & 105 & ra n. sp. . & & & & 111 \\
\hline unicolor Macq. . & $\Rightarrow$ & - & 105 & Scyphella pumilio n. sp. & - & & & 113 \\
\hline ». xanthogaster Wied. & • & & 106 & Sepsis javanica n. sp. . & & & & 107 \\
\hline Megaspis crassus $\mathrm{F}$. & - & & 100 & Tinda indica Walk. & & & & 96 \\
\hline zonalis F. & & & 100 & Tipula thibetana n. sp. & & & & 89 \\
\hline Microchrysa flaviventris Wied. & . & & 94 & monochroa Wied: . & & & & 89 \\
\hline Microdon metallicus n. sp. & & & 98 & praepotens Wied. & & & & 89 \\
\hline Milesia gigas Macq. . . & • & & 99 & Volucella ursina n. sp. . & & & & 101 \\
\hline ulva $\mathrm{r}$ & & & 99 & Xenaspis vespoides n. sp. . & & & & 107 \\
\hline Musca modesta n. sp & & & 106 & Zygaenula albofasciata n. sp.. & & & & 108 \\
\hline
\end{tabular}




\section{TAFELERKL Ä RUNG.}

Fig. 1. Pselliophora stigmatican. sp.

Fig. 2. Tipula thibetana n. sp. Flügel.

Fig. 3. " 》. Hinterleibsende.

Fig. 4. Pachyrrhina dimidiata n. sp. Thorax.

Fig. 5. Dicranom yia Kobusi klügel.

Fig. 6. 》" $\gg$ Krallenglied (Praetarsus).

Fig. 7. Rhipidia pulchra n. sp.

Fig. 8. Hermetia laeta n. sp.

Fig. 9. $"$ fenestratan. sp.

Fig. 10. Eudmeta brunnean. sp.

Fig. 11. Ptecticus tricolor n. sp.

Fig. 12. Ptilocera quadridentata F. Schuppenförmiges Haar vom Thorax.

Fig. 13. Rosapha bi maculata n. sp.

Fig. 14. " " Kopf.

Fig. 15. Chrysopila simplex n. sp. Discoidalzelle.

Fig. 16. Milesia sem ifulva n. sp. Hinterleib.

Fig. 17. Baccha refulgens Aust. Flügel.

Fig. 18. Sepsis javanica n. sp. Schenkel und Schiene des männlichen Vorderbeines.

Fig. 19. Xenaspis vespoides n. sp.

Fig. 20. - ” " Flügel.

Fig. 21. Zygaenula albofasciata n. sp.

Fig. 22. Rhabdochaeta pulchella n. sp. Flügel.

Fig. 23. " " Härchen des Flügels.

Fig. 24. Pseudeurina maculatan. sp.

Fig. 25. 》 $》$ Kopf.

Fig. 26. Myrmemorpha rufescens n. sp, Flügel.

Fig. 27. Hippelates bilineatus n. sp. Kopf.

Fig. 28. ” " Flügel.

Fig. 29. Scyphella pumilio n. sp. Flügel.

Fig. 30. Drosomyia picta n. sp. Kopf.

Fig. 31. ». " Flügel. 
PI. VIII.

Bijdragen tot de Dierkunde.
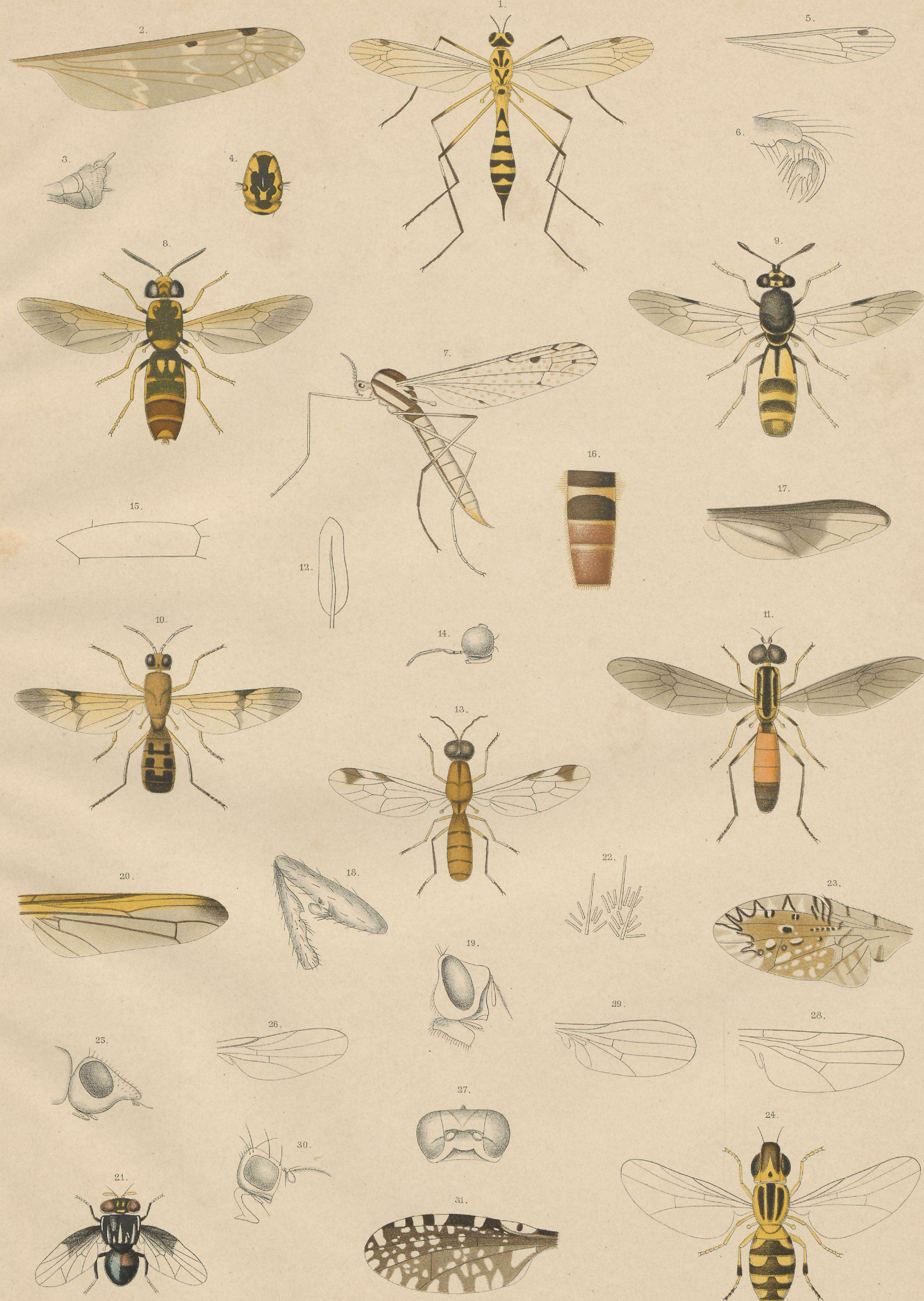